\title{
Hyperspectral measurements of yellow rust and fusarium head blight in cereal crops: Part 1: Laboratory study
}

\author{
Rebecca L Whetton ${ }^{\mathrm{a}}$, Kirsty L Hassall ${ }^{\mathrm{b}}$, Toby W Wainea and Abdul M Mouazen ${ }^{\mathrm{c*}}$ \\ aCranfield Soil and AgriFood Institute, Cranfield University, Bedfordshire MK43 0AL, UK. \\ ${ }^{b}$ Department of Computational and Analytical Sciences, Rothamsted research, Harpenden, \\ Hertfordshire AL5 2JQ \\ 'Department of Soil Management, Ghent University, Coupure 653, 9000 Gent, Belgium. \\ E-mail of corresponding author: Abdul.Mouazen@UGent.be
}

\begin{abstract}
This paper assesses the potential use of a hyperspectral camera for measurement of yellow rust and fusarium head blight in wheat and barley canopy under laboratory conditions. Scanning of crop canopy in trays occurred between anthesis growth stage 60 , and hard dough growth stage 87 . Visual assessment was made at four levels, namely, at the head, at the flag leaves, at $2^{\text {nd }}$ and $3^{\text {rd }}$ leaves, and at the lower canopy. Partial least squares regression (PLSR) analyses were implemented separately on data captured at four growing stages to establish separate calibration models to predict the percentage coverage of yellow rust and fusarium head blight infection. Results showed that the standard deviation between 500 and $650 \mathrm{~nm}$ and the squared difference between 650 and $700 \mathrm{~nm}$ wavelengths were found to be significantly different between healthy and infected canopy particularly for yellow rust in both crops, whereas the effect of water-stress was generally found to be unimportant. The PLSR yellow rust models were of good prediction capability for 6 out of 8 growing stages, a very good prediction at early milk stage in wheat and a moderate prediction at the late milk development stage in barley. For fusarium, predictions were very good for seven growing stages and of good performance for anthesis growing stage in wheat, with best performing for the milk development stages. However, the root mean square error of predictions for yellow rust were almost half of those for fusarium, suggesting higher prediction accuracies for yellow rust measurement under laboratory conditions.
\end{abstract}

\section{Key words}

Yellow rust (Puccinia striiformis), fusarium head blight (Fusarium graminearum), wheat, barley, crop canopy, partial least squares regression. 


\section{Introduction}

30 With the world's population estimated to reach 9 billion by 2050, sustainable approaches to increase crop yield are a necessity (Hole et al., 2005; Godfray et al., 2010). Current farming practices are unsustainable, relying on external inputs and high-yield varieties susceptible to disease (Hole et al., 2005). Site specific management of inputs would reduce the amount required (Wittery and Mallarino 2004; Maleki et al., 2007). Among these resources, fungicide application may well be reduced by targeted site specific spraying (FRAC 2010). However, accurate measurement of fungal diseases is a main requirement for sustainable application of fungicides, and expected to contribute to the reduction and prevention of the spread of crop disease and the losses of quantity and quality incurred from them.

Fungal disease control is a large task for a successful production of cereals worldwide. Both yellow rust and fusarium are fungal diseases which infect small cereal crops, and are responsible for causing severe yield losses (De Vallavieille-Pope et al., 1995; Bravo et al., 2003). Yellow rust caused by Puccinia striiformis is a foliar disease, which can reduce crop yields by up to $40 \%$. Alternatively known as stripe rust, the pathogen produces yellow uredo spores on the leaves. Infection starts with chlorosis occurring parallel to leaf veins, in a narrow $2 \mathrm{~mm}$ wide stripe, which develops later into multiple yellow between plants. In severe epidemics the yield can be reduced by up to 7 tonne ha-1 (Bravo et al., 2003). Fusarium head blight is one of the most important pre-harvest diseases worldwide, reducing yield quantity and quality. The most aggressive and prevalent fusarium strain is Fusarium graminearum, which is a highly pathogenic strain producing mycotoxins, which can become a significant threat to both humans and animals. Fusarium head blight symptoms in wheat and barley appear in the head and peduncle tissues, causing discolouration and early senescence. Disease presence can vary considerably between plants (Desjardin, 2006; Brennan et al., 2005; Leslie and Summerell, 2006; Rotter et al., 1996), hence, it is required to adopt site specific treatments of fungal diseases.

Advanced methods for disease detection in crops are vital for improving the efficacy of treatment, reducing infection and minimising the losses to yield and quality. Traditionally, disease detection is carried out manually, which is costly, time consuming and requires relevant expertise (Schmale \& Bergstrom, 2003; Bock et al., 2010a). Alternative methods of detection are needed to enable mapping the spatial distribution of yellow rust and fusarium head blight. Among those methods, optical sensing methods are recommended candidates since they are non-destructive and allow for fast and repeated data acquisition throughout the growing season without inhibiting crop growth. It was recognised by West et al., (2003) that although optical technologies are available for development into suitable disease 
61 detection systems, many challenges are still needed to be overcome, and this is still arguably the case.

62 Spectroscopy and imaging techniques have been used in disease and stress monitoring (Hahn, 2009).

63 One of the optical methods reportedly used to measure disease in crops is hyperspectral imaging in 64 the visible (vis) and/or the near infrared (NIR) spectral ranges. The reflectance at visible wavelength 65 range is relevant to leaf pigmentation whilst the infrared wavelength range provides information 66 on the physiological condition of the plant. The wavelength function for light intensity in 67 hyperspectral imaging adds to the brightness information of the spectral image, providing a rapid 68 image-contrast (Huang et al., 2007). Within the visible spectrum, the radiation reflectance from an 69 environmentally stressed plant will increase. This is due to an increase in the incidence reflection within the leaf of a stressed plant (Cibula and Carter, 1992). Bélanger et al.,(2008) showed that disease could be quantified on detached leaves, and reported that the ratio of blue (near $440 \mathrm{~nm}$ ) over green (near $520 \mathrm{~nm}$ ) intensities between the healthy and diseased tissue was significantly different shortly after inoculation. Using a vis-NIR imaging, Bravo et al.,(2003) detected early symptoms of yellow rust on winter wheat, with a quadratic discriminant model analysis, reporting a correct discrimination accuracy of $92-98 \%$. To our knowledge none of the above studies incorporated the effect of water stress, in the prediction model of yellow rust and fusarium head blight intensity in cereal crops. Some studies have focused on bringing the technology to the field. However, the first step towards field application is to test the accuracy of the methods under laboratory conditions (allowing more control and observation of the crop), where disease and water stress are accounted for simultaneously.

The aim of this paper is to assess the potential implementation and performance of a hyperspectral imager for recognition of yellow rust and fusarium head blight diseases in winter wheat and winter barley under laboratory conditions, with the intention to establish calibration models and a spectral library for potential use under mobile on-line measurement conditions. Both diseases (yellow rust and fusarium head blight) and water stress were introduced and accounted for.

\section{Materials and methods}

\subsection{Wheat and Barley cultivation and inoculation}

Treated seeds of winter wheat Triticum sativum (Solstice variety) and winter barley Hordeum vulgare L. (Carat Variety) were grown outdoors in 600 x $400 \mathrm{~mm}$ trays (depth of $120 \mathrm{~mm}$ ), with 100 seeds evenly sown and spaced in 5 parallel lines. After seeding the trays were predominantly rain fed, to reduce input of excess salts from treated tap water. Three treatments were adopted, where each treatment was triplicated in three separate trays. A total of 18 trays of wheat, and 18 trays of barley were grown for each of the following three treatments: 
94 1) Treatment 1 - Healthy: consisting of six trays of each that were kept healthy by applying a broad spectrum fungicide (Rubric and Epoxiconazole, at a rate of $1 l \mathrm{ha}^{-1}$ ).

2) Treatment 2 - Naturally (non-inoculated) yellow rust infected: consisting of six trays that were not treated with fungicide, as these were to represent the more heavily infected yellow rust trays, and were not inoculated with fusarium.

99 3) Treatment 3 - Fusarium inoculated: consisting of six trays of each that were infected with fusarium as the crop first reached anthesis growing stage (Figure 1).

When the crop growth reached 'booting' growth stage 45 on the Zadoks scale (Zadoks et al., 1974), half of trays in each treatment were water stressed using a transparent tarpaulin and water content was monitored throughout the growing season using a moisture-probe ML3 Thetakit (Delta-T Devices Ltd, Cambridge, UK). Yellow rust occurred naturally in the crops as early as growth stage 30 . Therefore, half of the crop trays were treated early with fungicide to fulfil treatments 2 and 3 . This allowed for a difference in intensity of yellow rust disease. Fusarium inoculation was applied to trays in treatment 3 at the anthesis crop growth stage. The spores were first cultivated in the laboratory by using the following method. A 2\% wheat agar was produced using $100 \mathrm{ml}$ distilled water, with $2 \mathrm{~g}$ agar and $2 \mathrm{~g}$ milled wheat. This was autoclaved at $120^{\circ} \mathrm{C}$. Plates were poured to a consistent depth, and inoculated with Fusarium graminearum. The plates were grown for 5-7 days under UV light as this was shown to help cause sporulation (Leach, 1967). The agar plates were subsequently agitated with distilled water to suspend the spores with the concentration increased as necessary by gentle use of the centrifuge. Spore concentrations were standardised at approximately $10^{6} \mathrm{ml}^{-1}$ using serial dilutions and a haemocytometer. Every $1 \mathrm{~m}^{2}$ of crop ear was inoculated with $100 \mathrm{ml}$ of the suspension, which is an adapted method from Lacey (1999). These trays were then kept under a high humidity conditions for 24 hours.

\subsection{Disease assessments}

A common approach for disease assessments and general crop health is by visual inspection known as diagnosis (Oberti et al., 2014 ). Chiarappa (1981) defined two distinct quantitative disease measurements: 1) Disease incidence, which is the percentage of infected plants to the healthy and 2) Disease severity, which is the amount of expressed disease tissue of a plant. These disease parameters can be assessed objectively, with some potential risk of subjectivity. In the current work, we considered the disease severity measured as \% coverage. Each tray was assessed for both diseases at 
four levels, namely, at the head (when present), at the flag leaves, $2^{\text {nd }}$ and $3^{\text {rd }}$ leaves (mid canopy), and at the lower canopy, as explained next;

1) For fusarium infection, only the head of the crop was assessed, since fusarium head blight symptoms in wheat and barley usually only appear in the head and peduncle tissues, causing discolouration and

\subsection{Hyperspectral data capture}

A push broom hyperspectral imager (spectrograph) (HS spectral camera model from Gilden Photonics Ltd., UK) was used to capture high-resolution (1,608 pixels) line images over 1 second, using a diode array detector. It is a 12 bit Basler piA 1600-35 gm camera, with Schneider-Kreuznach XNP1.4/23 lens and has a pixel pitch of $7.4 \mu \mathrm{m}$ interpolated/averaged to $0.6 \mathrm{~nm}$ readings with a spectral range of 400 $1000 \mathrm{~nm}$. The reflected light from the target travels through the lens, past an entrance slit through a series of inspector optics in the spectrograph and then split by the prism dispersing element into different wavelengths. This sensor was chosen for its potential for being applied to crop canopy measurements, and was of a lower price compared to comparable sensors, commercially available in the market. 
The spectral data was captured at three separate places along the crop tray at slightly different positions. Captured in the form of a line array, each pixel has a spectrum and one detector per pixel across the swath. In order to compile a full image, every line across a target must be captured (Gilden Photonics Ltd, Glasgow, UK). When configured on a consistent moving platform, the imager sweeps across an area to build up an image. Due to practical constraints of applying a consistent moving platform, the spectraSENS v3.3 (Gilden Photonics Ltd, Glasgow, UK) software was adapted to record a single line array, which required an additional RGB photo taken by a 5 megapixel camera with a $3.85 \mathrm{~mm} \mathrm{f} / 2.8$ lens at the same time of image capture, so that the scanned area could be comprehended. Two laser pointers were added at each side of the hyperspectral imager to indicate the area of the canopy to be scanned (Figure 3). The laser pointers were shut off when the spectral image was captured to remove any interference. The collected scans were corrected by means of a dark and a white reference (spectralon 99\% white reflectance panel) providing the relative reflectance. The latter was used before spectral capture, and at 10 minute intervals until scanning was completed. The optimal configuration of the push broom hyperspectral imager including light sources was optimised in the laboratory (Whetton et al., 2016). A schematic illustration of the configurations can be observed in Figure 3, where two 500 watt diffused broad spectrum halogen lamps were positioned at either end of the crop sample tray. Light angle was kept constant at $45^{\circ}$, which is suggested as the optimal angle to provide the strongest response (Huadong, 2001). The optimal configuration adopted included integration time, light height, light distance, camera height, and camera angle, of $50 \mathrm{~ms}, 1.2 \mathrm{~m}, 1.2 \mathrm{~m}$, $0.3 \mathrm{~m}$ and $10^{\circ}$, respectively (Whetton et al., 2016). These configurations were used in the current work, for crop canopy scanning that started at booting growth stage 60 on Zadok's scale and continued until reaching ripening at growth stage 87 . Four scans collected at four growth stages are considered in this study for both wheat and barley: 1) at anthesis (GS 60), 2) at kernel development; early milk (GS 72), 3) at kernel development; late milk (GS 77), and 4) hard dough (GS 87) (Table 1).

\subsection{Data pre-processing and modelling}

If the spectral data are too noisy there is a risk that key features of the spectrum are hidden, which necessitates smoothing to remove noise. But, aggressive smoothing can also remove significant features (Dasu \& Johnson, 2003), hence the need for a gentle smoothing to avoid losing of useful spectral features. Furthermore, a noisy spectrum can result in poor model performance, due to noise being considered a feature. Thus, the first step towards successful measurement should be to obtain a good quality spectrum. This was ensured in the current work by adopting the optimal configurations established in Whetton et al., (2016). The three lines of captured spectral data from each tray at each 
time were averaged first, before they were linked with the visual crop assessment. The spectral range outside of the 400 to $750 \mathrm{~nm}$ range was removed as it was noisy. The first and last 320 pixels from each line scan were removed due to variation and risk of overlapping the crop to the surrounding background. Both these pre-processing steps of the data are in line with Whetton et al., (2016). The spectral data was averaged to reduce the number of wavelengths (variables), which was successively followed by maximum normalisation, Savitzky-Golay first derivative and smoothing (Mouazen et al., 2006). Maximum normalisation is typically used to get all data to approximately the same scale, or to get a more even distribution of the variances and the average values. The maximum normalisation is a normalisation that "polarizes" the spectra. The peaks of all spectra with positive values scale to +1 , while spectra with negative values scale to -1 . Since all soil spectra in this study have positive values, the peaks of these spectra scaled to +1 . This scaled spectra between 0 and +1 . Using the SavitzkyGolay first derivative enables the computation of the first or higher-order derivatives, including a smoothing factor, which determines how many adjacent variables will be used to estimate the polynomial approximation used for derivatives. A second order polynomial approximation was selected. A 2:2 smoothing was carried out after the first derivative to decrease noise from the measured spectra. All pre-processing steps were carried out using Unscrambler 10 software (Camo Inc.; Oslo, Norway).

Analysis of variance (ANOVA) was used to analyse two spectral indices captured at growth stage 72. A factorial treatment structure was incorporated to test for differences between disease type (healthy, fusarium, yellow rust), water treatment (watered, water-stressed) and crop type (barley, wheat). In addition, a contrast was used to test for differences between healthy and diseased trays and between the different diseases. Analysis of the index SD was done on a log scale, whilst analysis of SQdiff was done on a sqrt scale to ensure homoscedascity of variance. GenStat $18^{\text {th }}$ Edition (C) VSN International Ltd, Hemel Hempstead, UK) was used to compute the ANOVA tables.

Principal component analysis (PCA) was used to investigate the multivariate hyperspectral response over the different scanning intervals for barley and wheat data separately. The first two principal components accounted for $92 \%$ of the variation in both the barley and wheat data. Consequently, for both crops, PCA provides a reasonable summary of the hyperspectral response in two dimensions.

Separate PLSR analyses were applied to each of the four scanning intervals to establish quantitative models to predict yellow rust and fusarium head blight infection (Table 1). This means that for each crop four PLSR analyses were carried out. Before PLSR analysis, data were divided into two sets of $80 \%$ (e.g., 43 samples) and 20\% (e.g., 11 samples), representing the calibration and prediction 
data sets (Tables 2 and 3), respectively. The pre-processed spectra and visual assessments of yellow rust and fusarium head blight of the calibration dataset were subjected to PLSR with leave-one-out full cross-validation to establish calibration models. The performance of these models was evaluated by predicting crop disease using the prediction dataset. Separate models for wheat and barley were developed and evaluated for yellow rust and fusarium head blight. The following models were developed and validated:

229 1) Yellow rust prediction in wheat and barley, estimated as \% of disease symptoms spread on the leaves. This was referred to as yellow rust $\%$ coverage.

2) Fusarium head blight prediction in wheat and barley, estimated as \% of infected ears. This was referred to as fusarium $\%$ coverage.

233 For both models, a logit transformation of the \% coverage response was applied to ensure 234 homoscedascity of variance. The inverse LOGIT function $(\exp (\mathrm{p}) /(1+\exp (\mathrm{p}))$ was applied before 235 assessment of the prediction results. PLSR analysis was carried out using Unscrambler 10 software 236 (Camo Inc.; Oslo, Norway). Outliers were detected, and removed to a maximum of 5\% of the total 237 input data. The model performance was evaluated in cross-validation and prediction by means of coeefecient of determination $\left(R^{2}\right)$, root mean square error of prediction (RMSEP) and ratio of prediction deviation (RPD), which equals standard deviation divided by the RMSEP. In order to compare between the performances of the developed models we proposed classifying RPD values into the classes mentioned in Table 4. The entire pre-processed spectrum was used in both the PCA and PLSR analyses.

\section{$243 \quad 3$ Results and discussion}

\subsection{Crop canopy spectra}

246 Example of crop canopy spectra for wheat and barley are shown in Figure 4. The spectral signatures were selected to demonstrate clearly the variations in shape. An arrows have been added to highlight wavelengths that define spectrum regions containing the most visible variation between the two crops. In Figure 4, wheat has higher reflectance. This may be due to the particular spectrum selected, as generally the reflectance intensities of wheat and barley were witnessed to be similar. However, it may also be attributed to the larger leaf area of wheat, which reflected more light than barley, which has smaller surface area. Within the visible range of $400-550 \mathrm{~nm}$, there is low reflectance due to larger 
absorption of the light, attributed to the photosynthetic pigments of the plant leaves, governed by the abundance of chlorophyll, which absorbs most of the light radiation (Gates et al., 1965; Thomas \& Gausman, 1977). Both plant chlorophylls and carotenoids have strong absorption at $480 \mathrm{~nm}$, the waveband associated with blue colour (Hunt et al., 2013). Another interesting band at $670 \mathrm{~nm}$ (associated with red colour band at $680 \mathrm{~nm}$ ) can be linked with chlorophyll a absorption that also appears at $550 \mathrm{~nm}$ (Hunt et al., 2013). The latter wavelength is designated as the green leaf reflectance (Buscaglia and Varco, 2002 and Zhao et al., 2005). The strongest absorption wavelength band appears at the red edge around $715 \mathrm{~nm}$, with deeper absorption in the barley spectrum than in wheat. Raper and Varco (2015) found that the strongest wavelength correlations with leaf nitrogen concentration, yield and plant total nitrogen content are near $700 \mathrm{~nm}$. Further analysis of these bands as linked with crop diseases studied is discussed below.

Average spectra of healthy, yellow rust and fusarium head blight infected wheat crop canopies at growth stage 72 are plotted in Figure 5. While plots a, b and c juxtapose irrigated and water stressed spectra, plot d compares between healthy and infected canopies under irrigated conditions. Generally, all spectra are similar, although slight differences can be observed by close examination of individual plots (Figure 5, b and c). The water-stressed spectra are less reflective than watered spectra, particularly for yellow rust (Figure 5a). Slight differences in spectral shape can be observed in the healthy canopy (a), which is in line with the findings from Earl and Davis (2003) who attributed these differences to alterations in leaf internal structure, variations in leaf angle (due to wilting) and leaf area index. Lower reflectance at the green edge $(500-570 \mathrm{~nm})$ and red edge $(670-750 \mathrm{~nm})$ can be attributed to water stress. However, these slight differences may indicate that water-stress has only slight influence on crop canopy, hence, on the performance of PLSR models in predicting yellow rust and fusarium head blight. The influence of water stress on yellow rust infected crop canopy is more obvious, where the waterstressed spectrum is consistently of lower reflectance (higher absorption) than the watered spectrum throughout the entire waveband (Figure 5b). This indicates that water stress may have a considerable influence on yellow rust prediction. However, spectra pre-processing e.g., maximum normalization used in this study will eliminate difference in reflectance e.g., due to scattering, as all spectra will be scaled between 0-1. Only a small deviation is observed between fusarium head blight infected spectra (Figure 5c), indicating little effect of water stress on fusarium head blight prediction. This is supported by the statistical analysis of the indices discussed below (Table 6).

A close examination of Figure 5d indicates notable differences in spectra between healthy, yellow rust and fusarium head blight infected crop canopies under watered conditions. The healthy spectrum is of lower reflectance than both infected spectra in the range between 400 to $700 \mathrm{~nm}$. This could be attributed to larger photosynthetic pigments of the plants associated with chlorophyll (Gates et al., 
1965; Thomas and Gausman, 1977). Cibula and Carter (1992) reported larger reflectance in infected leaves than healthy leaves, which is in line with findings of the current study. Indeed, after crop infection from foliar diseases, such as yellow rust, noteworthy visual symptoms can usually be observed. Early symptoms such as chlorosis, associated with a reduction in chlorophyll results in increasing reflectance due to a reduction in light absorption (Lorenzen and Jensen, 1989). Therefore, the sharpest increase in reflectance from 650 to $700 \mathrm{~nm}$ takes place in the healthy spectrum. Figure 6 compares between the average spectra of healthy, yellow rust and fusarium head blight infected barley canopy at growth stage 72 . The water-stressed canopy spectrum shows more reflection or less absorption than the watered canopy spectrum for the healthy canopy in Figure 6a. This may reflect the darker (greener) canopy of the watered canopy resulting in larger absorption of light. This is in line with findings of other researchers, who have attributed the increased reflectance of the healthy canopy to early senescence caused by drought, and a reduction in chlorophyll absorption (Jamieson et al., 1995; Hunt et al., 2013). With yellow rust infected canopy (Figure 6b), the opposite trend can be observed, where higher reflectance is shown for the water-stressed canopy. This trend is observed in both the wheat (Figure 5b) and barley (Figure 6b) canopies, indicating a larger influence of yellow rust on crop canopy when combined with water stress, compared to fusarium (Figures $5 \mathrm{c}$ and $6 \mathrm{c}$ ), where the differences between watered and water-stressed are minimal. As for wheat canopy, yellow rust infected canopy has again the highest reflectance, compared to those of fusarium head blight and healthy canopies (Figure 6d). The \% coverages of yellow rust and fusarium head blight is larger in wheat than in barley. In wheat, yellow rust watered canopy have an average infection of $42 \%$, yellow rust water stressed $45 \%$, fusarium watered $83 \%$, fusarium water stressed $86 \%$, whereas in barley, these are $36 \%, 33 \%, 48 \%$ and $52 \%$, respectively.

In order to quantify differences between healthy, yellow rust and fusarium head blight infected spectra two indices were taken into account in this study, namely, standard deviation (SD) of all wavelengths in the 500-650 nm range and squared difference (SQdiff) of 650 and $700 \mathrm{~nm}$ (Table 5). Moshou et al., (2004) recommended the use of wavelength range between 460 and $900 \mathrm{~nm}$ for successful yellow rust detection. Bauriegel (2011) recommends spectral analysis using the range intervals of 500-533 nm (green), 560-675 nm (yellow), 682-733 nm (red) and 927-931 nm (red edge) for recognition of Fusarium head blight infection (in growth stages 71-85, according to zadoks scale). Krishna, et al., (2014), suggested particularly useful spectra wavelengths of 428,672, and 1399, for quantitative detection of yellow rust from healthy crop.

318 These two proposed indices show clear differences in response both in the different crops and the different treatments. The largest differences are observed between infection type, a significant $F$ statistic 
of $\mathrm{F}_{1,24}=1199(\mathrm{p}<0.001)$ and $\mathrm{F}_{1,24}=33(\mathrm{p}<0.001)$ was observed for the comparison between fusarium infection and yellow rust infection, for index SD and SQdiff respectively.

Analysis of the index SD revealed significant differences in response in barley and wheat $\left(\mathrm{F}_{1,24}=94.59\right.$, $\mathrm{p}<0.001)$ and big differences between healthy and diseased trays $\left(\mathrm{F}_{1,24}=874.11, \mathrm{p}<0.001\right)$. The largest differences were observed between fusarium infection and yellow rust infection $\left(\mathrm{F}_{1,24}=1199.23\right.$, $\mathrm{p}<0.001)$. In contrast, there was no evidence of a significant main effect of water stress $\left(\mathrm{F}_{1,24}=1.79\right.$, $\mathrm{p}=0.193$ ), meaning that on average (over all disease types and crops) there is no evidence of a difference in the SD index for watered and water stressed trays. However, analysis of the index SD does demonstrate a significantly different response to water stress both within different crops and under different disease infections (full ANOVA table is given in Table 6), i.e. the response to water stress is not the same in the different conditions.

Analysis of the index SQdiff revealed significant differences between healthy and diseased trays $\left(\mathrm{F}_{1,24}=12.66, \mathrm{p}=0.002\right)$ and also significant differences between fusarium infection and yellow rust infection $\left(\mathrm{F}_{1,24}=33.29, \mathrm{p}<0.001\right)$. Moreover, different responses in the different crops was observed $\left(\mathrm{F}_{1,24}=7.61, \mathrm{p}=0.011\right)$ with a significant interaction between crop type and disease type indicating the index SQdiff responds differently to disease type in the different crops $\left(F_{1,24}=9.88, p=0.004\right)$. There was no evidence to suggest a differing response to water treatment $\left(\mathrm{F}_{1,24}=0.07, \mathrm{p}=0.799\right)$.

Although the largest SQdiff in reflectance between 650 and $700 \mathrm{~nm}$ is observed for the healthy canopy (both watered and water-stressed) of wheat, the smallest SD is observed for yellow rust (Table 5). For the barley canopy, the largest SD and SQdiff can be observed for fusarium head blight infected canopies, indicating that these proposed two indices respond differently for different crops (Table 5). Consequently, the two indices adopted in the current work highlight a distinguishable difference between the yellow rust, fusarium head blight and healthy wheat and barley crop canopies. It is important to mention that whilst these indices have worked in establishing a difference between yellow rust, fusarium and a healthy canopy at growth stage 72 in this paper, it may be specific to the method and equipment used. Further work should be undertaken to assess the reliability of such indices, if captured at different growth stages, under different circumstances, with alternative equipment. This is an important point to make as a strong correlation of time to spectral change was observed through PCA. The first two PCs (principal components) are shown in Figure 7 (for wheat) and figure 8 (for barley). The separation of observations in this two-dimensional representation is strongly associated with the time of scanning. Moreover, very little association with disease coverage could be discerned. This demonstrates that in the captured data when all timings are considered, the strongest influence on the canopies reflectance is time. These results supported the decision to split the scans per time of capture, for the PLSR of yellow rust and fusarium predictions. 


\subsection{Model performance for yellow rust detection}

The PLSR cross-validation and prediction results for yellow rust detection in are shown in Table 7. Separate PLSR were carried out for each time intervals of T1, T3, T5 and T7 for barley and T2, T4,

T6 and T8 for wheat (Table 1). The cross-validation results indicate good model performance for yellow rust $\%$ coverage in wheat and barley $\left(\mathrm{R}^{2}\right.$ values for wheat are $0.82,0.92,0.77$ and 0.84 , for T2, T4, T6 and $\mathrm{T} 8$ and barley $0.88,0.78,0.76$ and 0.83 for $\mathrm{T} 1, \mathrm{~T} 3, \mathrm{~T} 5$ and $\mathrm{T} 7$, respectively), showing low root mean square errors of cross-validation (RMSECV) ranging from 3.3 to $8.8 \%$. In general, the barley cross-validation results for yellow rust, have a slightly lower $R^{2}$ values and larger RMSECV than the corresponding values for wheat (Table 7). As yellow rust is a foliar disease, this reduction in prediction performance for barley may be attributed to the crop having a smaller flag leaf, and due to density of the crop, causing a smaller foliar area to be captured by the hyperspectral imager.

When the developed PLSR models where used to predict the yellow rust $\%$ coverage of $20 \%$ of samples (11 samples) in the prediction set, the RMSEP values in both wheat and barley show larger values in the predictions than in the cross-validations. However, RMSEP is a valuable index for assessing individual model performance, but is not recommended to compare the performance between different models (e.g., those for wheat and barley and between different growing stages), due to the different data range. To compare between the performances of different models, RPD was used in this work, according to the RPD classes proposed in the current work (Table 4). The RPD values for prediction of each timing (growth stage), shown in Table 7, suggest good prediction capability for 6 out of 8 growing stages (RPD ranges of 2.16-2.49 in wheat and 2.18-2.43 in barely), a very good prediction for T4 (kernel development, early milk (GS 72) in wheat $(\mathrm{RPD}=2.79)$ and a moderate prediction capability for T5 (kernel development; late milk (GS 77) in Barely (RPD) = 1.83).

It is well known in spectral analysis that successful measurement of a concentration, be it soil properties or other, depends on presence of variability of that said concentration. For example, Kuang and Mouazen (2011) reported that although larger $R^{2}$ and RPD can be obtained with larger variability in soil analysis, larger RMSEP is to be expected. Furthermore, with a small variability, weak or even no correlation can be established with PLSR, so that no models can be developed. Having said that, we believe that the scale of variability in \% coverage of yellow rust is rather small (Tables $2 \& 3$ ), although a reasonably high infection is recorded at few points (see the mean and SD values). The small variability may be due to the experiment being run in trays under rather controlled conditions, where only water is varied artificially. These controlled conditions may lead to small variability in yellow rust (Tables $2 \& 3$ ). The percentage of disease coverage which is a method discussed by Chiarappa 
387 (1981) and defined as "disease severity", is the amount of expressed disease tissue of a plant. This method can be objective, but is definitely not free of subjectivity. In the current study all assessments are made by the same individual, which decreases the between assessment variability due to the subjective nature of the measurement. The more spectral wavelength indices captured and accounted for, the greater understanding of the object (Gilchrist, 2006). However, for noisy spectra there is a need to minimise noise in the signal, by adopting an optimised measurement configuration (Whetton et al., 2016) and suitable spectra pre-processing. Furthermore, stresses in the field are combined and might include water stress, nitrogen stress, disease stress, and other stresses that are mainly reflected on crop canopy as a yellowing of the leaves. In the current work we have combined water stress and yellow rust infection in the tray experiments, to evaluate the prediction accuracy of the yellow rust models.

The results obtained in this study for yellow rust prediction encourage exploring the ultimate goal of the current study, which is on-line measurement of yellow rust in the field using the hyperspectral imager $(400-750 \mathrm{~nm})$. However, additional affecting parameters exist in the field on top of the water stress accounted for in the current study, and these should also be evaluated. Using wheat trays under glass house controlled conditions, Moshou et al. (2014) reported successful discrimination of waterstressed from healthy plants with $99 \%$ accuracy. Their approach was based on a combination of hyperspectral (460-900 nm) and fluorescence imagery and machine learning models. The early success in field studies for hyperspectral imager's detection of yellow rust disease such as Moshou et al. (2004) and Bravo et al. (2003) focused on the presence of yellow rust in the field, not necessarily the intensity. Typically disease recognition attempts with hyperspectral and multispectral imaging are targeted to leaves rather than the canopy (Bock et al., 2010b). Whilst recent attempts using lower cost solutions for disease quantification in wheat based on RGB images (Zhou et al., 2015) provided larger error margins. Compared to other studies the current work achieved moderate to very good accuracy based only on a relatively cost-effective hyperspectral camera in the visible range only. In addition, we have accounted for the effect of water stress in the experimental trial, hence, this effect was included in the PLSR prediction models.

\subsection{Model performance for fusarium head blight detection}

The cross-validation results for \% coverage of fusarium head blight indicate good model performance in both wheat and barley $\left(R^{2}\right.$ values for wheat are $0.84,0.89,0.81$ and 0.83 , for T2, T4, T6 and T8 and barley $0.95,0.83,0.75$ and 0.79 for $\mathrm{T} 1, \mathrm{~T} 3, \mathrm{~T} 5$ and $\mathrm{T} 7$, respectively), with RMSECV range of 8.6 to $29 \%$ in wheat and 14 to $25 \%$ in barley (Table 7). However these RMSECV ranges are higher than 
those calculated for yellow rust. The lowest $R^{2}$ for cross-validation was once again for the late milk stage. Due to the method of inoculation explained-above, there was little variability observed in fusarium head blight disease intensity per timing (growing stage). Although the relatively low variability recorded for fusarium, the cross-validation results for both wheat and barley indicate good model performances (Table 7).

The prediction results indicate larger RMSEP values for fusarium head blight (RMSEP $=7.9-16.1 \%$ for wheat and $10.4-15.1 \%$ for barley) are calculated than those for yellow rust (RMSEP $=7.2-8.8 \%$ for wheat and 7.2 - 8.1 for barley). However, for RPD, the opposite case is true. According to RPD values, good (for one growing stage) to very good (for three growing stages) predictions are recorded for fusarium in wheat, whereas very good predictions are calculated for the four growing stages in barley (Table 7). Also, higher RPD values are calculated for the prediction of fusarium head blight in both crops. The lower RMSEP values calculated for yellow rust than those for fusarium suggest higher prediction accuracy for yellow rust (smaller error). This means that yellow rust can be detected with higher accuracy than fusarium head blight, an observation to be taken into account for future variable rate applications or relevant fungicides.

Fusarium head blight symptoms appear on crop heads at a late stage in the crop growing season (normally only after anthesis, but potentially at head emergence), allowing for limited number of scans to be collected. Bauriegel et al. (2011) claimed that fusarium head blight can be detected by spectral analysis in the spectral range of 400-1000 nm, with an identification accuracy of $87 \%$. These authors advised that the ideal timing for measurement at the medium milk stage (growth stage 75), though the scans were based on the crop ears against a black background. Delwiche et al. (2011) successfully differentiated between healthy kernels from fusarium head blight infected, reporting a 95\% classification accuracy. The results reported in the current study support the previous findings, as the highest prediction performance is recorded for the kernel development stages, at both the early and late milk. Bauriegel et al. (2011) have also reported the highest measurement accuracy of fusarium in the milk kernel development stage. However, the relatively lower RPD scores in the earlier scans (T1 for barley and T2 for wheat), may be attributed to a smaller standard deviation of the data sets (Tables 2 and 3 ).

In order to account for the temporal dependence in observations over the different scanning intervals collected at the four growing stages in this study (Table 1), it was necessary to run a separate PLSR analysis for each growing stage. This has resulted in a rather small number of samples for each PLSR analysis (e.g., 43 and 11 for the calibration and prediction sets, respectively). Therefore, it is necessary to consider a larger dataset in the PLSR analysis in a future work, and to explore new methods of data 
analysis based on machine learning and/or image processing, or adopt a modelling approach that can explicitly account for temporal dependence/repeated measures structure. It is also suggested to adopt a data fusion approach of both spectra and images, which is expected to provide more reliable model prediction performance. However, the results reported in this work are successful and encouraging to suggest testing the proposed hyperspectral technique in the visible range of 400-750 nm, coupled with PLSR as a potential tool for on-line measurement of the named two fungal diseases. However, there are other affecting parameters in the field than water stress that should be accounted for, which include within field variability in soil properties, varying ambient light, sensor-to-crop canopy height and angle.

\section{Conclusions}

The study explored the potential of a hyperspectral line imager $(400-750 \mathrm{~nm})$ for the detection of yellow rust and fusarium head blight in wheat and barley, based on partial least squares regressing (PLSR) analysis. The experiment was carried out in the laboratory under partially controlled environmental conditions where water stress effect was introduced. The results reported allowed the following five main points to be concluded:

1) The standard deviation of the wavelength range from 500 to $650 \mathrm{~nm}$ and the squared difference between $650 \mathrm{~nm}$ and $700 \mathrm{~nm}$ are of interest in discrimination between healthy, from yellow rust or fusarium head blight infected wheat and barley canopy.

2) The principle component analysis run on canopy spectral data collected on healthy, yellow rust and fusarium infected crops at multiple growth stages, reveal temporal pattern and time serial autocorrelations, which suggested the need for separate PLSR for each growing stage.

3) The best PLSR prediction performance for yellow rust in wheat was at the early milk of the kernel development stage, whereas for barley the best performance was at the anthesis and the early milk stages.

4) The best PLSR prediction performance for fusarium was at both the early and late milk of the kernel development stages in both wheat and barley.

5) Although higher ratio of prediction deviations were calculated for fusarium head blight, the smaller root mean square error of prediction for yellow rust suggested more accurate measurement of the latter under laboratory conditions. 
483 The laboratory trials in this study have been designed to emulate a field. The data used in the models 484 was all collected from the wheat and barley trays, designed to simulate a field canopy, so the variance 485 of reflectance due to canopy is included in the models. Whilst other properties such as illumination 486 angle, view positions, shadows, plant species, maturity and phenology can be controlled under 487 laboratory conditions, these parameters will have considerable influences under field conditions, which need to be evaluated with a future work planned in Part 2 of this study.

\section{Acknowledgement}

We acknowledge the funding received for FarmFUSE project from the ICT-AGRI under the European 492 Commission's ERA-NET scheme under the 7th Framework Programme, and the UK Department of 493 Environment, Food and Rural Affairs (contract no: IF0208). The corresponding author acknowledges the FWO funded Odysseus SiTeMan Project (Nr. G0F9216N).

\section{References}

Andersen AL. (1948). The development of Gibberella zeae head blight of wheat. Phytopathology 38:595-611.

499 Bauriegel, E., Giebel, A., Geyer, M., Schmidt, U. and Herppich, W.B. (2011). Early detection of 500 Fusarium infection in wheat using hyper-spectral imaging.Computers and Electronics in Agriculture, 50175 (2), pp.304-312.

502 Bélanger M.C., Roger J.M., Cartolaro P., Viau A.A., Bellon-Maurel V., Detection of powdery mildew 503 in grapevine using remotely sensed UV-induced fluorescence, Int. J. Remote Sens., 29 (2008), pp. 1707504172

505

512 Bravo, C., Moshou, D., West, J., McCartney, A., \& Ramon, H. (2003). Early disease detection in wheat 513

Bock, C.H., Poole, G.H., Parker, P.E. and Gottwald, T.R. (2010a). Plant disease severity estimated visually, by digital photography and image analysis, and by hyperspectral imaging. Critical Reviews in Plant Sciences,29(2), pp.59-107

Bock, C. H., Graham, J. H., Gottwald, T. R., Cook, A. Z., \& Parker, P. E. (2010b). Wind speed effects on the quantity of Xanthomonas citri subsp. citri dispersed downwind from canopies of grapefruit trees infected with citrus canker. Plant Disease, 94(6), 725-736. fields using spectral reflectance. Biosystems Engineering, 84(2), 137-145. 
Brennan, J.M., Egan, D., Cooke, B.M, Doohan, F.M. (2005). Effect of temperature on head blight of wheat caused by Fusarium culmorum and F. graminearum. Plant Pathology 54, 156.

Buscaglia, H. J., \& Varco, J. J. (2002). Early detection of cotton leaf nitrogen status using leaf reflectance. Journal of Plant Nutrition, 25, 2067-2080.

Chiarappa L., ed. (1981). Crop Loss Assessment Methods - Supplement 3. Wallingford; CAB Interationa Cibula WG, Carter GA. (1992). Identification of a far-red reflectance response to ectomycorrhizae in slash pine. International Journal of Remote Sensing, 13:925-32.

Dasu, T., \& Johnson, T. (2003). Exploratory data mining and data cleaning (Vol. 479). John Wiley \& Sons.

Delwiche, S.R., Kim, M.S. and Dong, Y. (2011). Fusarium damage assessment in wheat kernels by Vis/NIR hyperspectral imaging. Sensing and instrumentation for food quality and safety, 5(2):63 71.

Desjardin, A.E. (2006). Fusarium mycotoxins. Chemistry, Genetics, and Biology. APS Press, St. Paul, MN.

de Vallavieille-Pope, C., Huber, L., Leconte, M. and Goyeau, H. (1995). Comparative effects of temperature and interrupted wet periods on germination, penetration, and infection of Puccinia recondita f. sp. tritici and P. striiformis on wheat seedlings. Phytopathology, 85(4), pp.409-415.

Earl, H.J. and Davis, R.F. (2003). Effect of drought stress on leaf and whole canopy radiation use efficiency and yield of maize. Agronomy journal, 95(3), pp.688-696.

Fungicide Resistance Action Committee, (2010). FRAC Recommendations for Fungicide Mixtures Designed to Delay Resistance Evolution.

Gates, D.M., Keegan, H.J., Schleter, J.C. and Weidner, V.R. (1965). Spectral properties of plants. Applied optics, 4(1): 11-20.

Gilchrist J. R. (2006). Gilden Photonics Ltd and Timo Hyvärinen, Spectral Imaging Ltd. Hyperspectral Imaging Spectroscopy: A Look at Real-Life Applications. Photonics.com. [Online].

Godfray, H.C.J., Beddington, J.R., Crute, I.R., Haddad, L., Lawrence, D., Muir, J.F., Pretty, J., Robinson, S., Thomas, S.M. and Toulmin, C., (2010). Food security: the challenge of feeding 9 billion people. science, 327(5967), pp. 812-818.

Goswami RS, Kistler HC. (2004). Heading for disaster: Fusarium graminearum on cereal crops. Mol Plant Pathol 5:515-525

Hahn, F., (2009). Actual pathogen detection: Sensors and algorithms - a review. Algorithms 2, 301338.

Hole, D., Perkins, A., Wilson, J. D., Alexander, I. H., Grice, P. V., \& Evans, A. (2005). Does organic farming benefit biodiversity? Biological Conservation, 122, pp. 113-130.

Home Grown Cereal Authority (HGCA) (2008). The spring wheat disease management guide. Warwickshire: Home Grown Cereals Authority (HGCA Publication). 
Huadong, G. (2001). Applications of Radar Remote Sensing in China. New York: Taylor and Francis inc. 56.

Huang, W., Lamb, D.W., Niu, Z., Zhang, Y., Liu, L. and Wang, J. (2007). Identification of yellow rust in wheat using in-situ spectral reflectance measurements and airborne hyperspectral imaging. Precision Agriculture,8(4-5), pp.187-197.

Hunt, E.R., Doraiswamy, P.C., McMurtrey, J.E., Daughtry, C.S., Perry, E.M. and Akhmedov, B., 2013. A visible band index for remote sensing leaf chlorophyll content at the canopy scale. International Journal of Applied Earth Observation and Geoinformation, 21, pp.103- 112

Impey, L. (2012). Fusarium found on wheat leaves in western England. Available: http://www.fwi.co.uk/articles/19/07/2012/134000/fusarium-found-on-wheat-leaves-in-western england.htm. Last accessed 14/10/2013

Jamieson, P.D., Martin, R.J., Francis, G.S. and Wilson, D.R., 1995. Drought effects on biomass production and radiation-use efficiency in barley. Field Crops Research, 43(2), pp.77-86.

Krishna, G., Sahoo, R.N., Pargal, S., Gupta, V.K., Sinha, P., Bhagat, S., Saharan, M.S., Singh, R. and Chattopadhyay, C. (2014). Assessing wheat yellow rust disease through hyperspectral remote sensing. The International Archives of Photogrammetry, Remote Sensing and Spatial Information Sciences, $40(8)$, p.1413.

Kuang, B.; Mouazen, A.M. (2011). Calibration of a visible and near infrared spectroscopy for soil analysis at field scales across three European farms. European Journal of Soil Science, 62(4): 629636.

Lacey J, Bateman GL, Mirocha CJ. (1999). Effects of infection time and moisture on the development of ear blight and deoxynivalenol production by Fusarium species in wheat. Ann Appl Biol 134:277-283.

Leach, C. M. (1967). Interaction of near-ultraviolet light and temperature on sporulation of the fungi Alternaria, Cercosporella, Fusarium, Helminthosporium, and Stemphylium. Canadian Journal of Botany, 45(11), 1999-2016.

Leslie, J.F., Summerell, B.A. (2006). The Fusarium Laboratory Manual. Blackwell Publishing, Ames, IA

Lorenzen, B. and Jensen, A., (1989). Changes in leaf spectral properties induced in barley by cereal powdery mildew. Remote Sensing of Environment, 27(2), pp.201-209.

Maleki, M.R., Mouazen, A.M., Ramon, H. and De Baerdemaeker, J., (2007). Optimisation of soil VIS-NIR sensor-based variable rate application system of soil phosphorus. Soil and Tillage Research, 94(1), pp.239-250.

McMullen MP, Jones R, Gallenberg G (1997). Scab of wheat and barley: a re-emerging disease of devastating impact. Plant Dis 81:1340-1348

Moshou, D., Bravo, C.,West, J.,Wahlen, S., McCartney, A., Ramon, H. (2004). Automatic detection of 'yellow rust' in wheat using reflectance measurements and neural networks. Computers and Electronics in Agriculture 44 (3), 173-188. 
Moshou, D., Pantazi, X.E., Kateris, D. and Gravalos, I. (2014). Water stress detection based on optical multisensor fusion with a least squares support vector machine classifier. Biosystems Engineering, 117, pp.15-22.

Mouazen, A., De Baerdemaeker, J. \& Ramon, H. (2006). Effect of wavelength range on the measurement accuracy of some selected soil constituents using visual-near infrared spectroscopy. Journal of Near Infrared Spectroscopy, Volume 14, p. 189-199.

Oberti, R., Marchi, M., Tirelli, P., Calcante, A., Iriti, M., \& Borghese, A. N. (2014). Automatic detection of powdery mildew on grapevine leaves by image analysis: Optimal view-angle range to increase the sensitivity. Computers and Electronics in Agriculture, 104, 1-8.

Parry DW, Jenkinson P, McLead L. (1995). Fusarium ear blight (scab) in small grain cereals- a review. Plant Pathol 44:207-238

Raper T. B. and Varco J. J. (2015) Canopy-scale wavelength and vegetative index sensitivities to cotton growth parameters and nitrogen status. Precision Agriculture, 16(1), pp 62-76)

Rotter, B.A., Prelusky, D.B., Pestka, J.J. (1996). Toxicology of deoxynivalenol (vomitoxin). Journal of Toxicology and Environmental Health 48, 1-34.

Schmale, D.G., III, \& Bergstrom, G.C. (2003). Fusarium head blightin wheat. The Plant Health Instructor. http://dx.doi.org/10.1094/PHI-I-2003-0612-01.

Thomas, J.R. and Gausman, H.W. (1977). Leaf reflectance vs. leaf chlorophyll and carotenoid concentrations for eight crops. Agronomy journal,69(5), pp.799-802

West, J.S., Bravo, C., Oberti, R., Lemaire, D., Moshou, D. and McCartney, H.A. (2003). The potential of optical canopy measurement for targested control of field crop diseases. Annu. Rev. Phytopathol, 41, pp.593-614.

Whetton, R.L., Waine, T.W. and Mouazen, A.M. (2017). Optimising configuration of a hyperspectral imager for on-line field measurement of wheat canopy. Biosystems engineering, 155, pp.84-95.

Wittry, D.J. and Mallarino, A.P., (2004). Comparison of uniform-and variable-rate phosphorus fertilization for corn-soybean rotations. Agronomy Journal, 96(1), pp.26-33.

Zadoks, J.C.; T.T. Chang, C.F. Konzak (1974). "A decimal code for the growth stages of cereals. Weed Research 14 (6): 415-421. doi:10.1111/j.1365-3180.1974.tb01084.x.

Zhao, D., Reddy, K. R., Kakani, V. G., Read, J. J., \& Koti, S. (2005). Selection of optimum reflectance ratios for estimating leaf nitrogen and chlorophyll concentrations of field-grown cotton. Agronomy Journal, 97, pp. 89-98.

Zhou, B., Elazab, A., Bort, J., Vergara, O., Serret, M.D. and Araus, J.L. (2015). Low-cost assessment of wheat resistance to yellow rust through conventional RGB images. Computers and Electronics in Agriculture, 116, pp.20-29. 


\section{Figure captions}

633 Figure 1: Fusarium inoculation of wheat and barley trays in the laboratory. Inoculation took place at the anthesis crop growth stage.

635 Figure 2: Illustrating influence of foliar health on yield (HGCA, 2008). The weight given in this study 636 was as follows; flag leaf $55 \%$, mid canopy $40 \%$, and lower canopy $5 \%$. This allowed a single yellow rust assessment to be associated to a tray.

638 Figure 3: Schematic illustration of the laboratory configurations of hyperspectral camera and light 639 source (Whetton et al., 2016).

640 Figure 4: Example spectra of wheat and barley canopy, after white and dark corrections.

641 Figure 5: Comparison of an average wheat crop canopy (growth stage 72) spectra between watered 642 (-) and water-stressed (----) treatments for healthy (a), yellow rust infected (b) and fusarium infected (c) crop canopy. Panel d compares canopy spectra under watered conditions of healthy (---), yellow rust (---) and fusarium (-).Watered yellow rust had an averaged infection of $42 \%$, water stressed yellow $45 \%$, watered fusarium $83 \%$, and water stressed fusarium $86 \%$.

646 Figure 6: Comparison of an average barley crop canopy (growth stage 72) spectra between watered (-) and water-stressed (----) treatments for a) healthy , b) yellow rust infected and c) fusarium infected 648 crop canopy. Panel d compares canopy spectra under watered conditions of healthy (---), yellow rust (---) and fusarium (-). Watered yellow rust had an average infection of 36\%, water stressed yellow rust $33 \%$, watered fusarium $48 \%$, and water stressed fusarium $52 \%$.

Figure 7: Principal component analysis (PCA) similarity map of wheat canopy spectral data determined by principal components 1 (PC1) and 2 (PC2), showing separation of different spectra collected at Timing 2 (T2) of anthesis growth stage 60, T4 of early milk growth stage 72, T6 of late milk growth stage 77, and T8 of hard dough growth sage 87 .

655 Figure 8: Principal component analysis (PCA) similarity map of barley canopy spectral data 656 determined by principal components 1 (PC1) and 2 (PC2), showing separation of different spectra 
657 collected at Timing 1 (T1) of anthesis growth stage 60, T3 of early milk growth stage 72 , T5 of late 658 milk growth stage 77, and T7 of hard dough growth sage 87. 


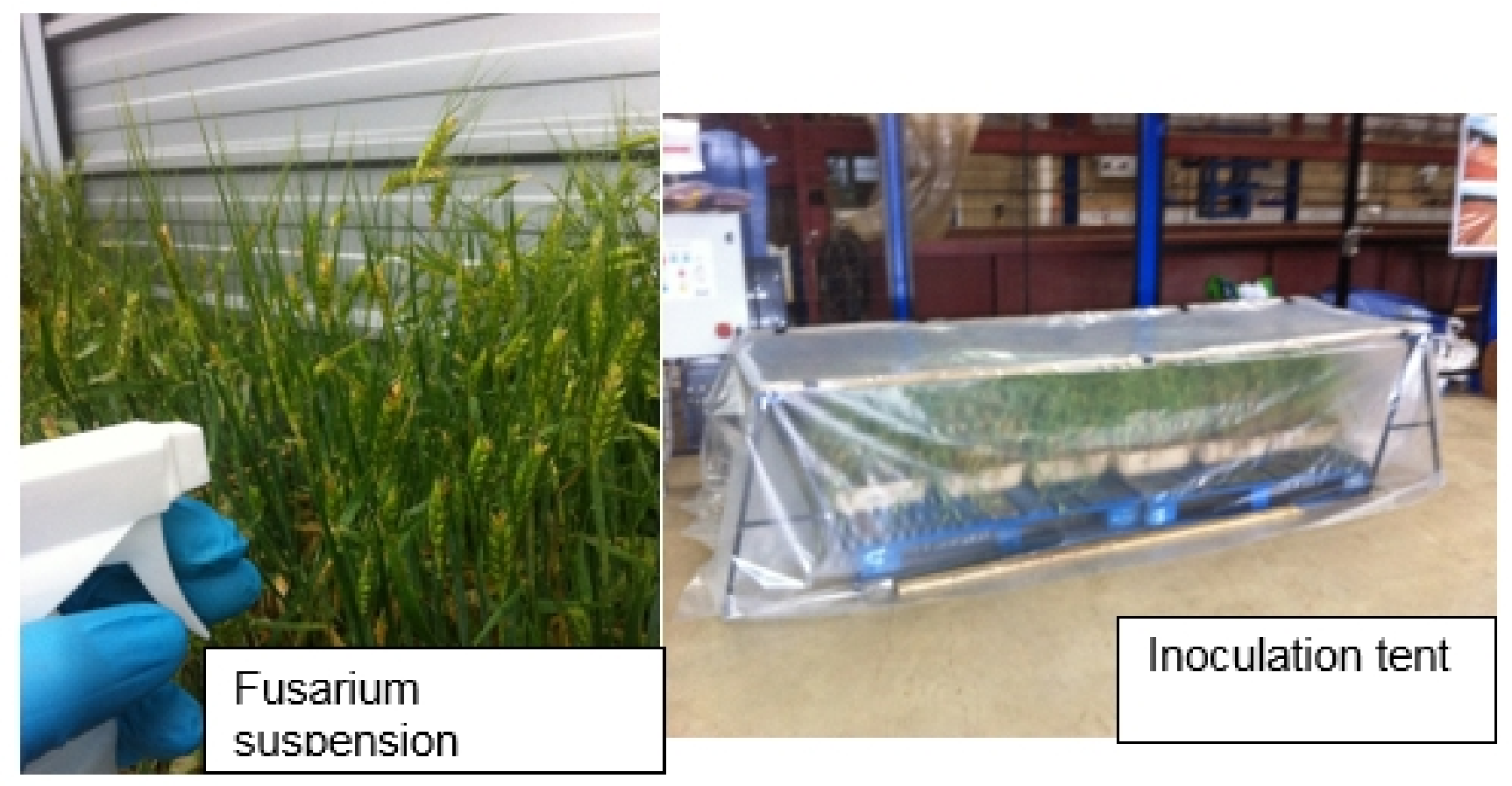




\section{$80 \%$ of wheat yield comes from the top three leaves}

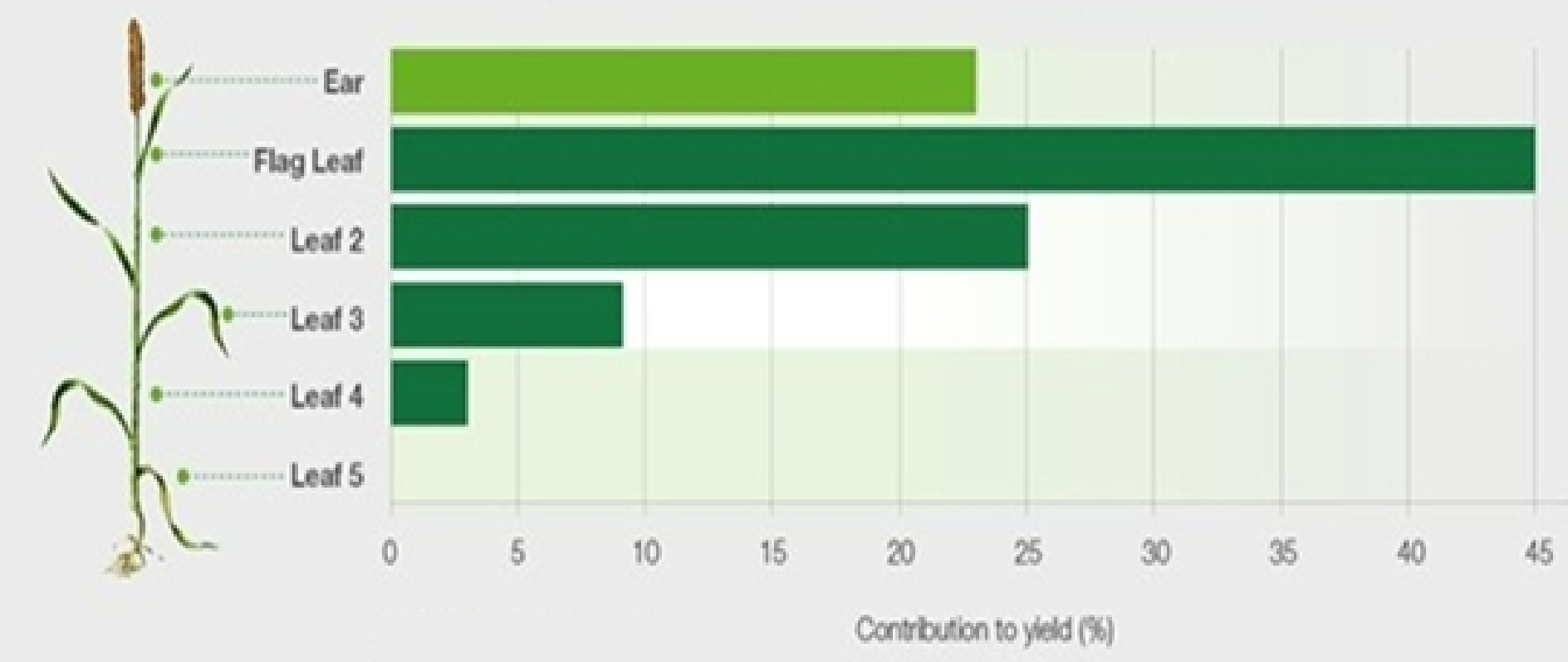




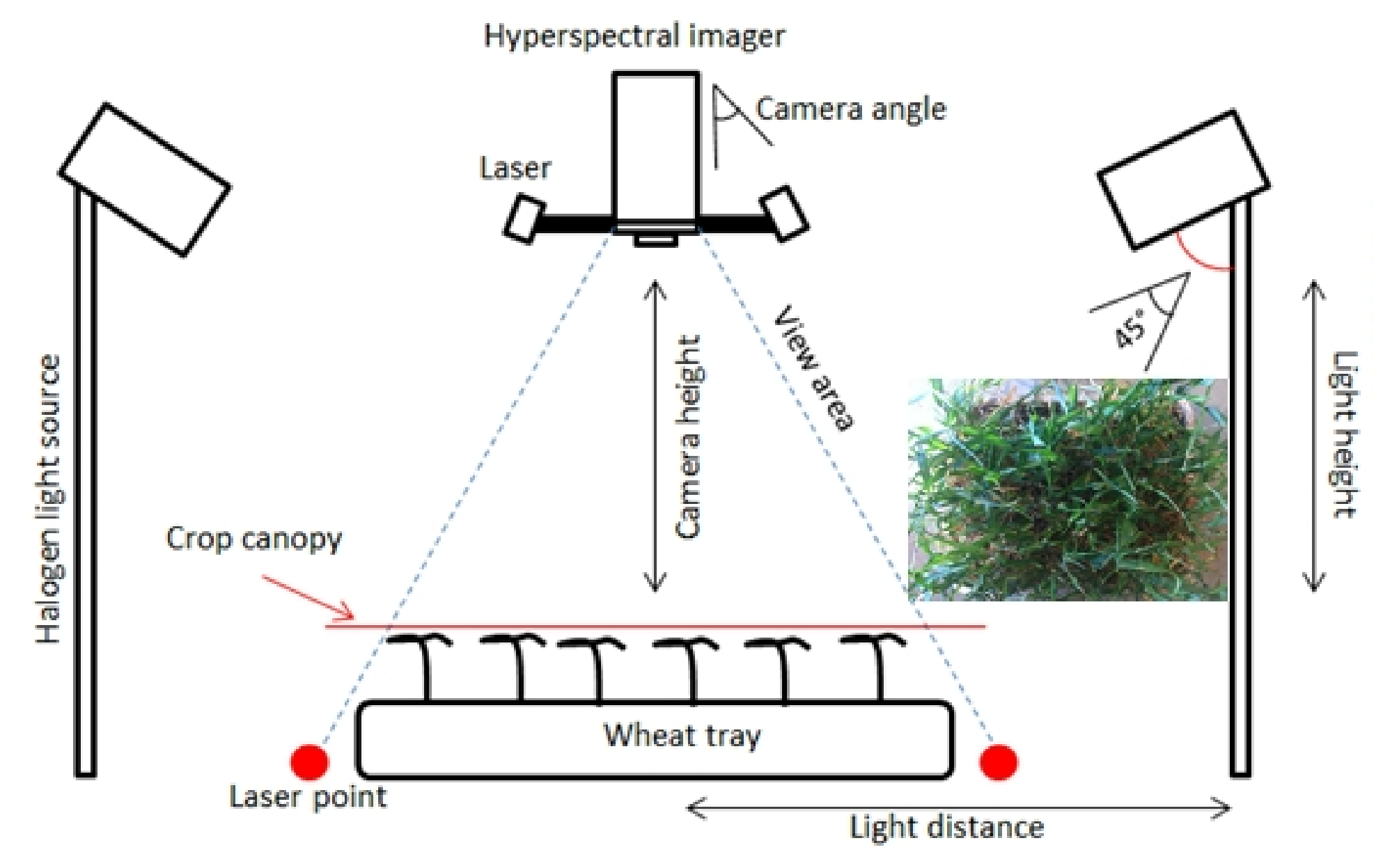




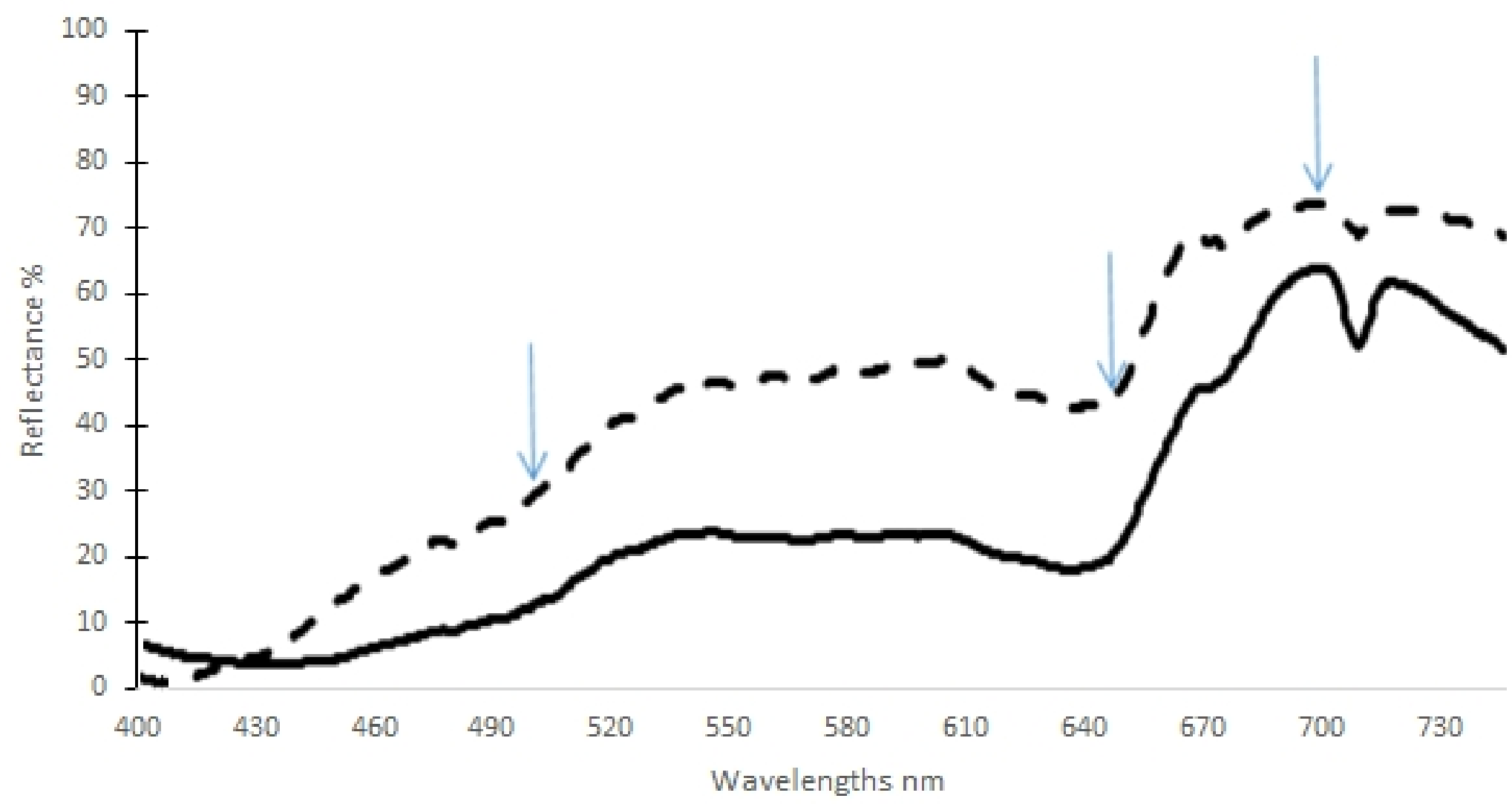

- - wheat 


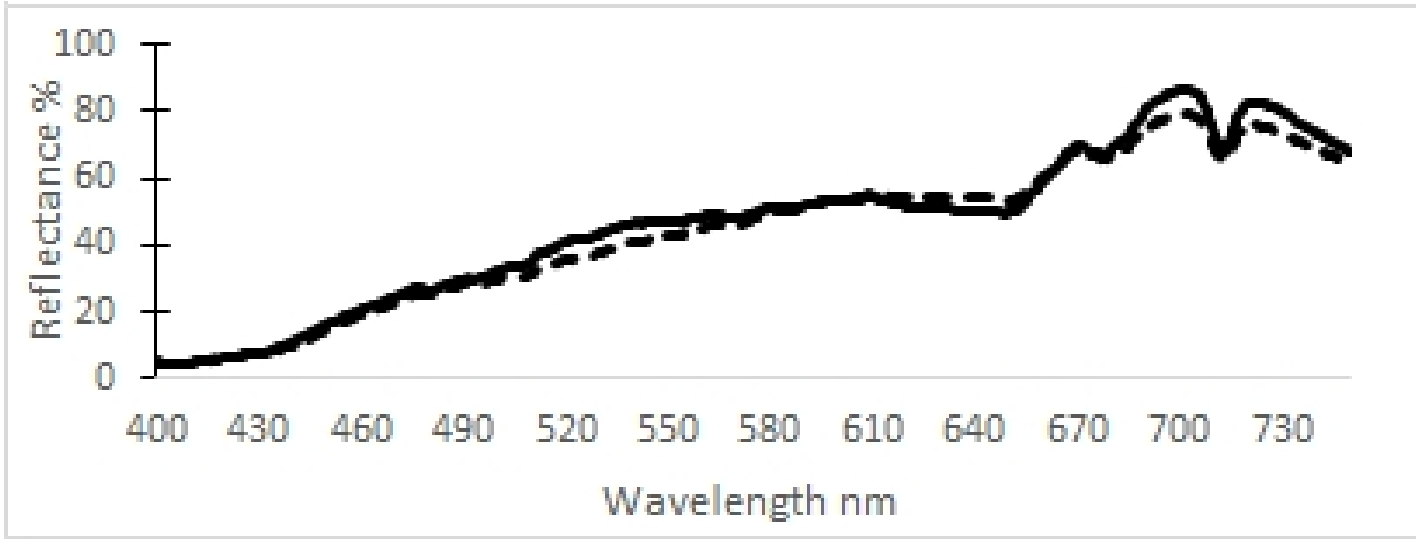

a)

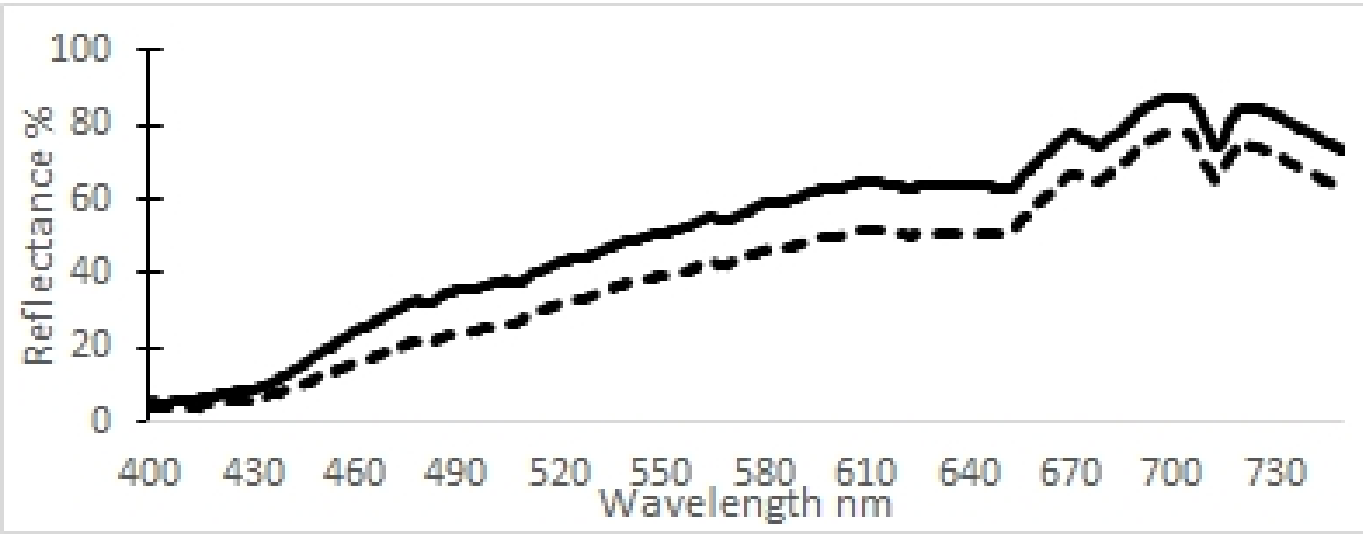

b)

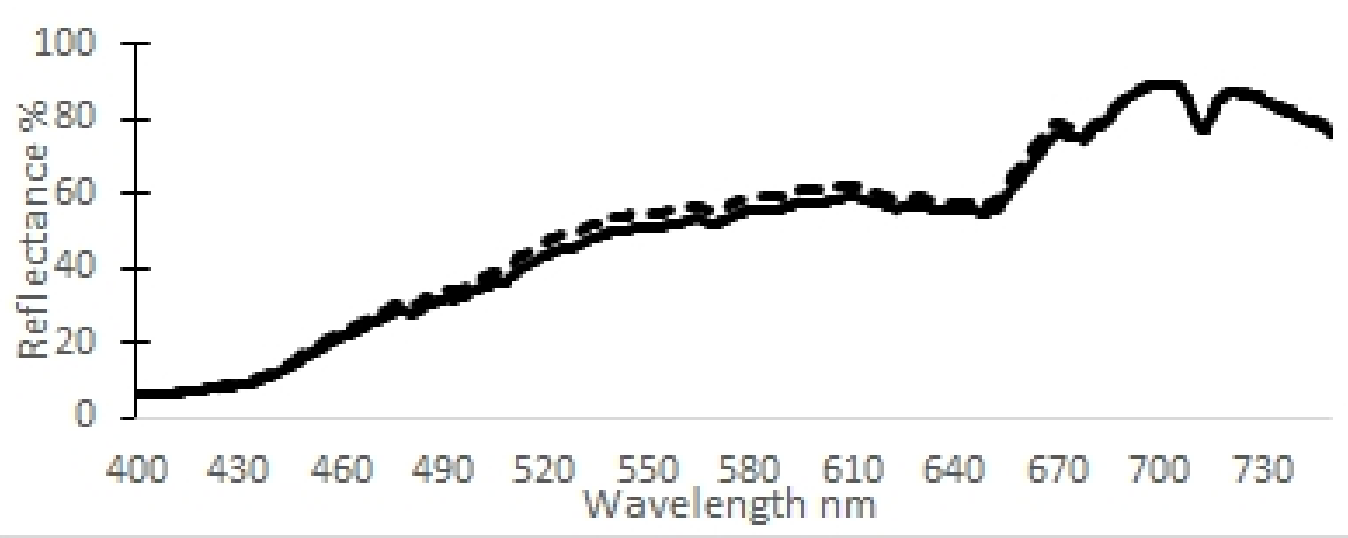

c)

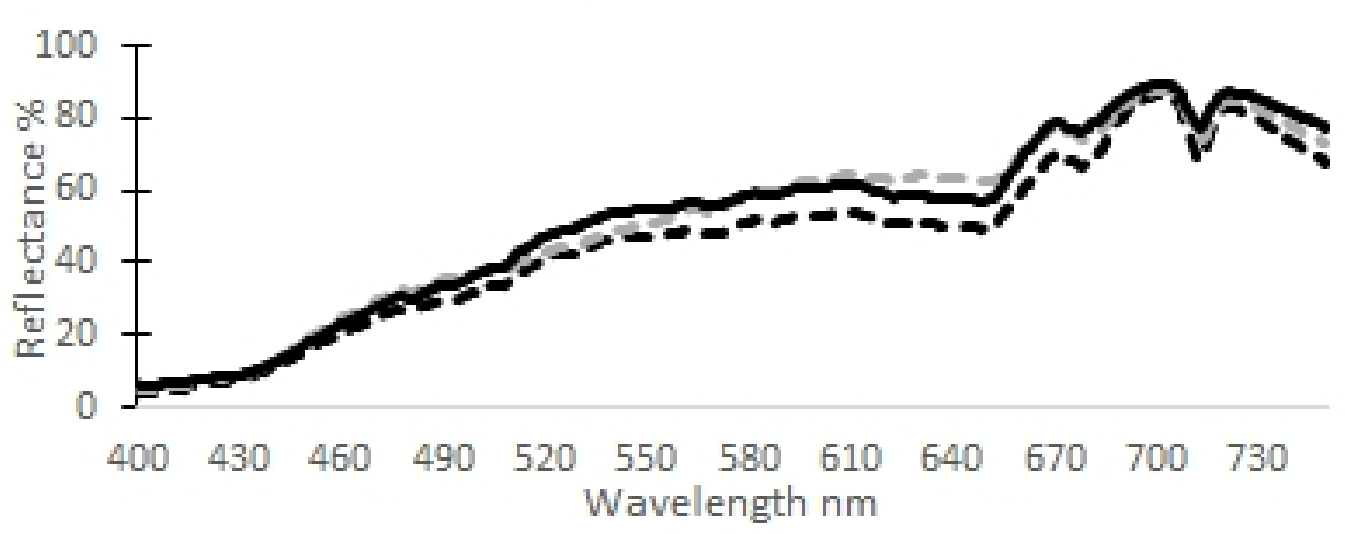

d) 


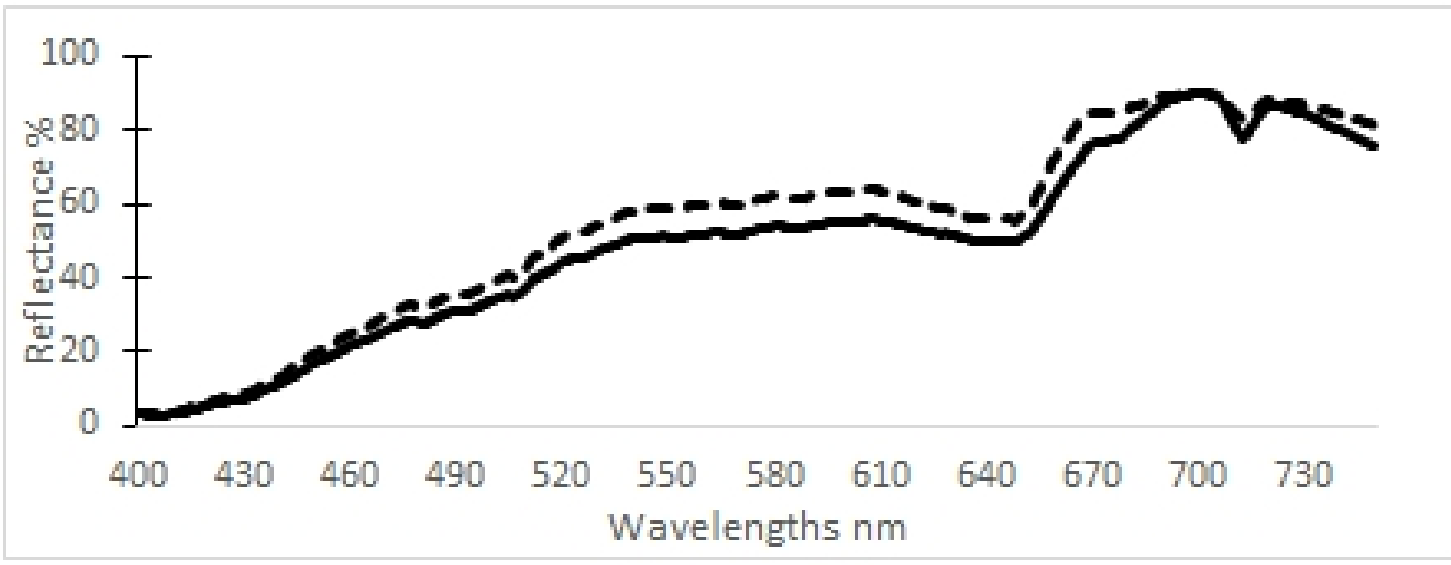

a)

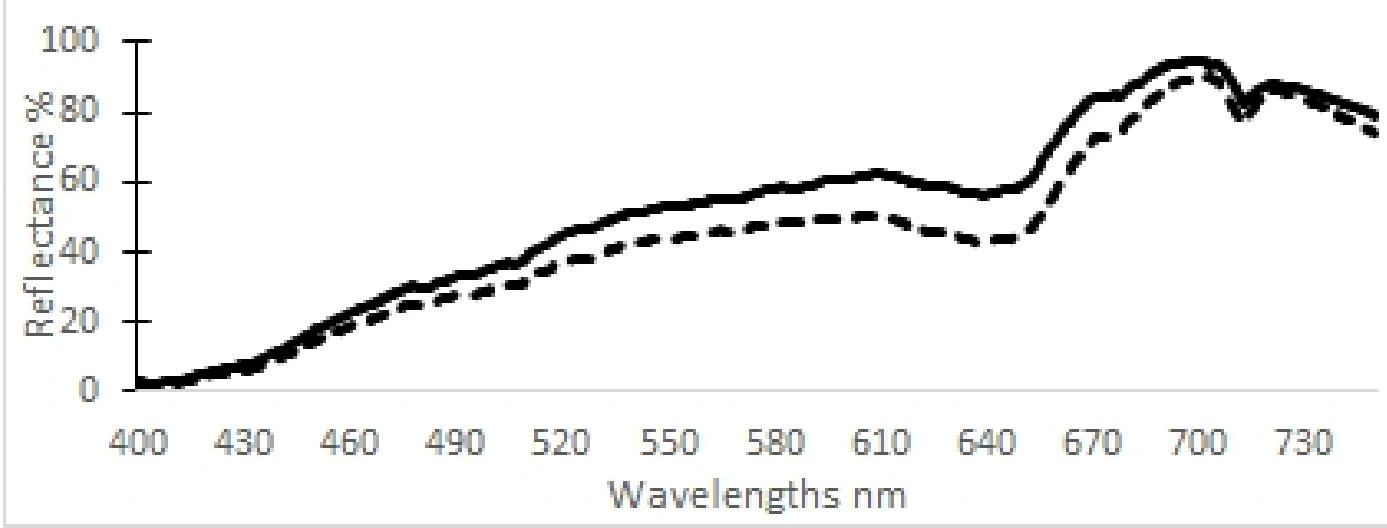

b)

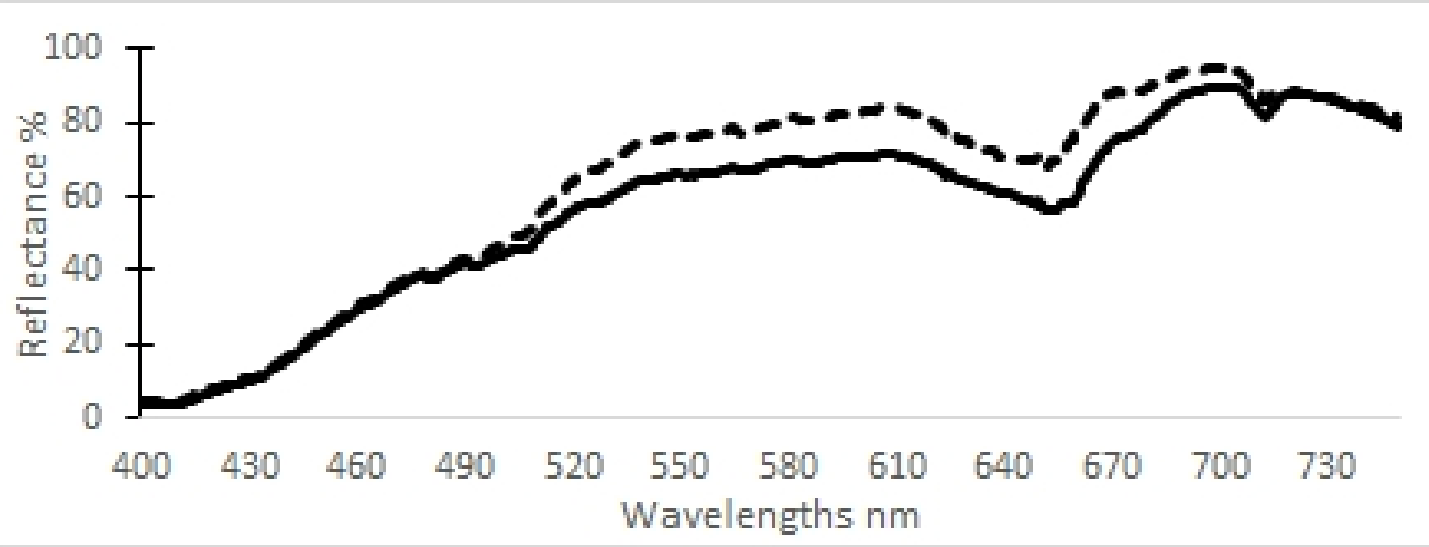

c)

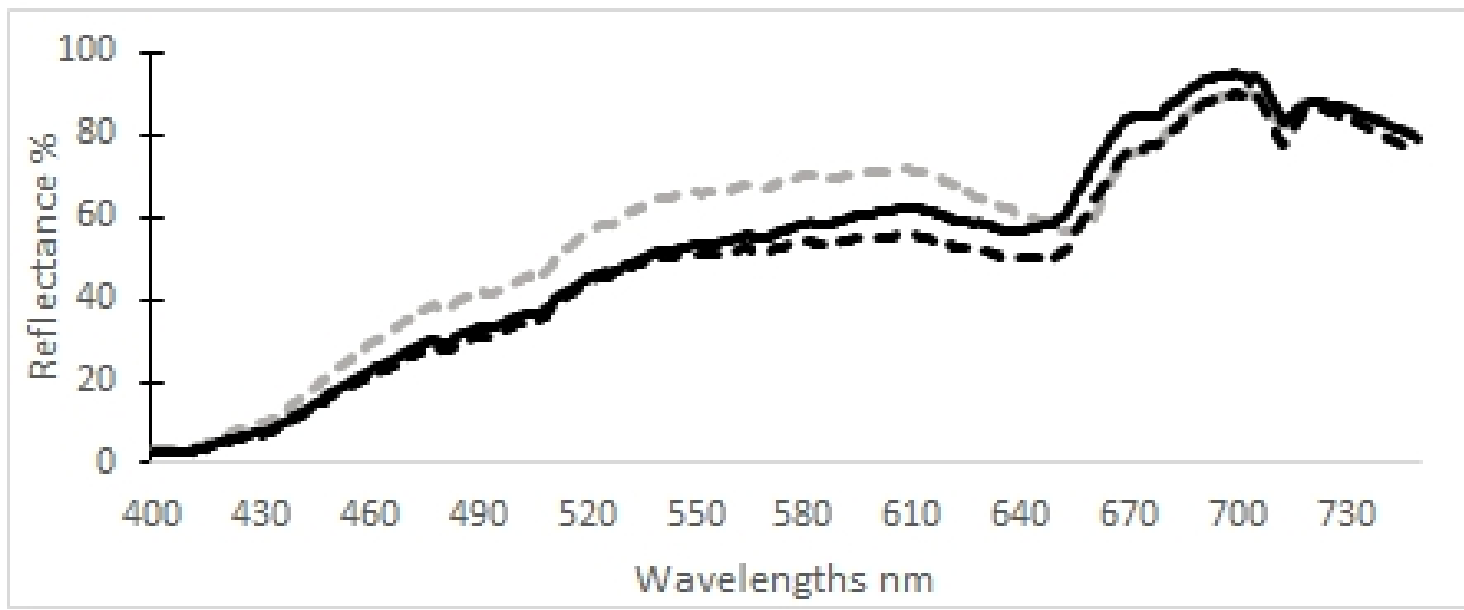

d) 


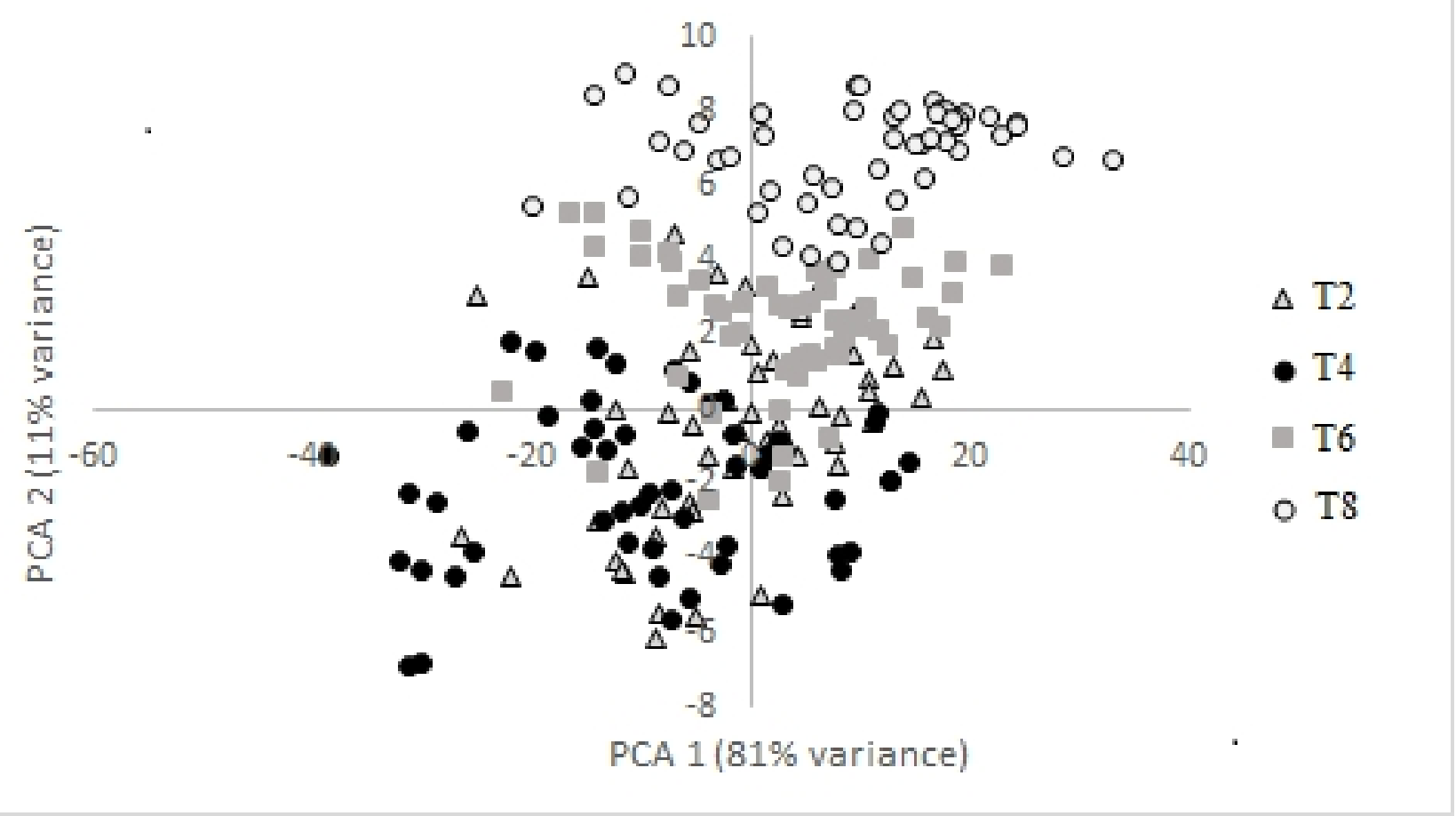




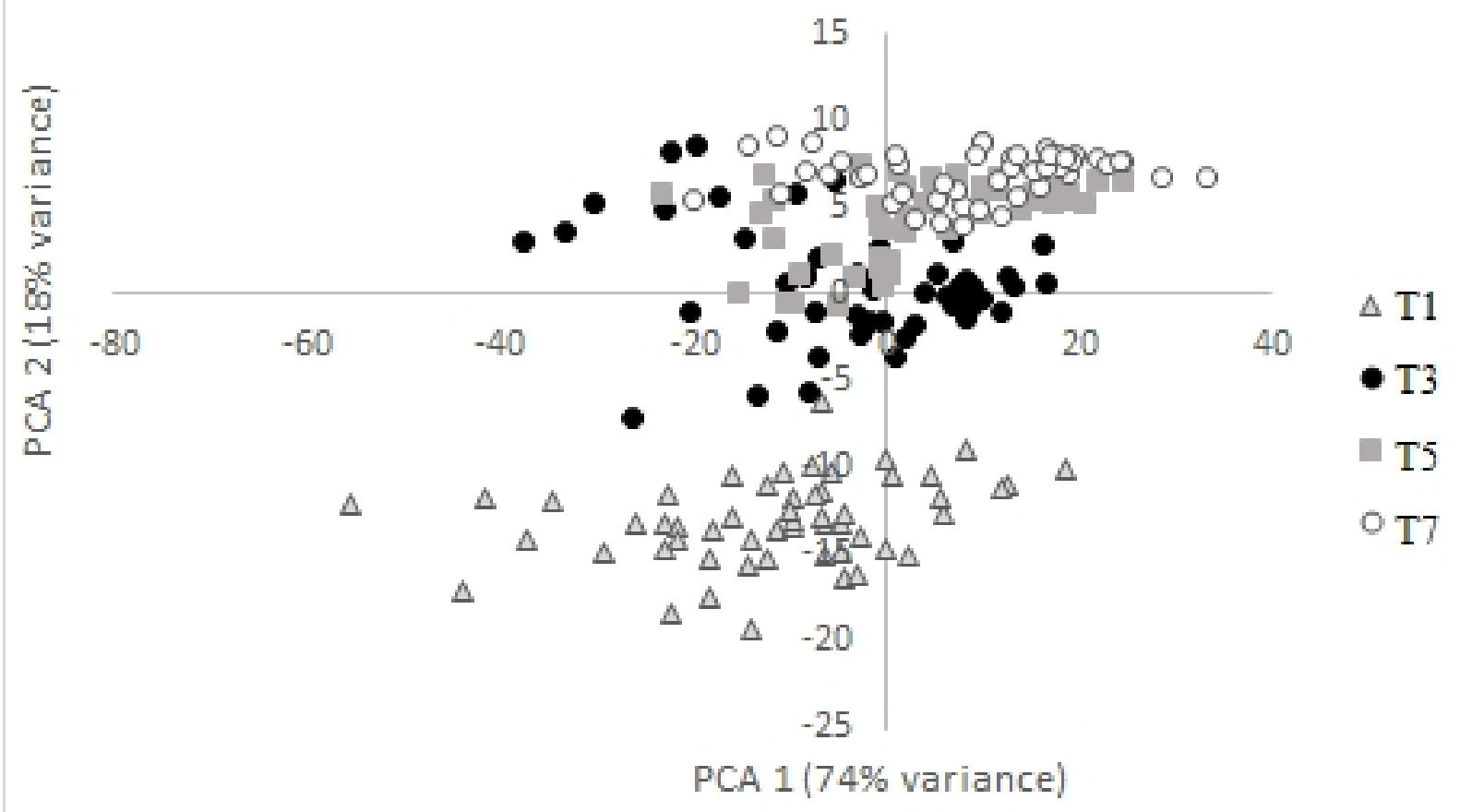


Table 1: Hyperspectral scanning intervals of the wheat and barley trays, at four growth stages (GS) according to Zadoks scale (Zadoks et al., 1974).

Timing Growth stage

\begin{tabular}{lll}
\hline Barley & 1 (T1) & Anthesis (GS 60) \\
& $3(\mathrm{~T} 3)$ & Kernel development; early milk (GS 72) \\
$5(\mathrm{~T} 5)$ & Kernel development; late milk (GS 77) \\
7 (T7) & Hard dough (GS 87) \\
\hline \multirow{2}{*}{ Wheat } & 2 (T2) & Anthesis (GS 60) \\
& $4(\mathrm{~T} 4)$ & Kernel development; early milk (GS 72) \\
& $6(\mathrm{~T} 6)$ & Kernel development; late milk (GS 77) \\
$8(\mathrm{~T} 8)$ & Hard dough (GS 87) \\
\hline
\end{tabular}


Table 2: Statistics of $\%$ coverage of both fungal diseases of wheat samples used in the partial least squares regression (PLSR) analyses, with $80 \%$ and $20 \%$ of samples were considered for cross-validation and prediction, respectively, at four separate timings (growth stages).

\begin{tabular}{|l|rrrr|rrrr|}
\cline { 2 - 9 } \multicolumn{1}{c|}{} & \multicolumn{3}{c|}{ Yellow rust } & \multicolumn{4}{c|}{ Fusarium } \\
\cline { 2 - 9 } \multicolumn{1}{c|}{} & $\mathrm{T} 2$ & $\mathrm{~T} 4$ & $\mathrm{~T} 6$ & $\mathrm{~T} 8$ & $\mathrm{~T} 2$ & $\mathrm{~T} 4$ & $\mathrm{~T} 6$ & $\mathrm{~T} 8$ \\
\hline Cross- & & & & & & & & \\
validation & & & & & & & & \\
Sample Nr. & 43 & 43 & 43 & 43 & 43 & 43 & 43 & 43 \\
Maximum (\%) & 70 & 65 & 55 & 40 & 55 & 100 & 100 & 100 \\
Minimum (\%) & 0 & 0 & 0 & 0 & 0 & 0 & 0 & 0 \\
Mean (\%) & 30.4 & 20.8 & 17.4 & 15.9 & 17.5 & 24.1 & 30.1 & 31.5 \\
SD (\%) & 21.4 & 11.8 & 11 & 11.3 & 23.0 & 32.4 & 43.2 & 45.0 \\
\hline Prediction & & & & & & & & \\
Sample Nr. & 11 & 11 & 11 & 11 & 11 & 11 & 11 & 11 \\
Maximum (\%) & 70 & 70 & 50 & 60 & 50 & 100 & 100 & 100 \\
Minimum (\%) & 0 & 10 & 5 & 0 & 0 & 0 & 0 & 0 \\
Mean (\%) & 33.6 & 30 & 19.4 & 17.9 & 12 & 40 & 47 & 34 \\
SD (\%) & 20.1 & 26.1 & 19 & 16.3 & 20.4 & 47.6 & 49.7 & 44.5 \\
\hline
\end{tabular}

SD is standard deviation; T2 is anthesis growth stage 60; T4 is early milk growth stage 72 ; T6 is late milk growth stage 77; and $\mathrm{T} 8$ of hard dough growth sage 87 in wheat. 
Table 3: Statistics of \% coverage of both fungal diseases in barley samples used in the partial least squares regression (PLSR) models, with $80 \%$ and $20 \%$ of samples were considered for cross validation and prediction, respectively, at four separate timings (growth stages).

\begin{tabular}{|l|llll|lllll|}
\cline { 2 - 9 } \multicolumn{1}{c|}{} & \multicolumn{3}{ll}{ Yellow rust } & & Fusarium & & \\
\cline { 2 - 9 } \multicolumn{1}{c|}{} & $\mathrm{T} 1$ & $\mathrm{~T} 3$ & $\mathrm{~T} 5$ & $\mathrm{~T} 7$ & $\mathrm{~T} 1$ & $\mathrm{~T} 3$ & $\mathrm{~T} 5$ & $\mathrm{~T} 7$ \\
\hline Cross \\
validation
\end{tabular}

SD is standard deviation; T1 is anthesis growth stage 60; T3 is early milk growth stage 72 ; T5 is late milk growth stage 77; T7 is hard dough growth sage 87 in barely. 
Table 4: Classes of the ratio of prediction deviation (RPD) and their suitability for predicting yellow rust and fusarium head blight in cereal crops, and is based on the classifications..

\begin{tabular}{lll}
\hline RPD range & Class and prediction capability & Prediction Category \\
\hline$<1$ & Poor model predictions - not useful. & A \\
$1-1.5$ & Possibility to discriminate between low and high values & B \\
$1.5-2.0$ & Moderate prediction capability & $\mathrm{C}$ \\
$2.0-2.5$ & Good prediction capability & $\mathrm{D}$ \\
$2.5-3.0$ & Very good prediction capability & $\mathrm{E}$ \\
$>3.0$ & Excellent prediction capability & $\mathrm{F}$ \\
\hline
\end{tabular}


Table 5: Spectral differences indicated as standard deviation (SD) of the 500-650 $\mathrm{nm}$ range and squared difference (SQdiff) of 650 and $700 \mathrm{~nm}$, calculated on the maximum normalised spectra for healthy, yellow rust, and fusarium infected wheat and barley canopies under watered and water-stressed conditions.

SD 500-650

(nm) (nm)

Wheat

$\begin{array}{lll}\text { Yellow rust watered } & 0.089 & 0.062 \\ \text { Yellow rust water-stressed } & 0.081 & 0.076 \\ \text { Healthy watered } & 0.057 & 0.15 \\ \text { Healthy water-stressed } & 0.063 & 0.14 \\ \text { Fusarium watered } & 0.16 & 0.10 \\ \text { Fusarium water-stressed } & 0.15 & 0.11\end{array}$

Barley

\begin{tabular}{lll} 
Yellow rust watered & 0.056 & 0.08 \\
Yellow rust water-stressed & 0.061 & 0.077 \\
Healthy watered & 0.051 & 0.15 \\
Healthy water-stressed & 0.065 & 0.18 \\
Fusarium watered & 0.15 & 0.25 \\
Fusarium water-stressed & 0.13 & 0.18 \\
\hline
\end{tabular}


Table 6: Analysis of Variance (ANOVA) tables for the analysis of transformed spectral indices over the different treatments. Analysis of the index the squared difference of 650 and $700 \mathrm{~nm}$ (sqDiff) was done on the square root scale, whilst analysis of the index standard deviation (SD) is done on of the range $500-650 \mathrm{~nm}$.

\begin{tabular}{|c|c|c|c|c|c|}
\hline \multicolumn{6}{|l|}{$\log (\mathrm{SD})$} \\
\hline & d.f. & s.s. & m.s. & v.r. & F pr. \\
\hline Disease Status (Healthy vs Infected) & 1 & 7.48442 & 7.48442 & 874.11 & $<.001$ \\
\hline Water (Watered vs Water stressed) & 1 & 0.015325 & 0.015325 & 1.79 & 0.193 \\
\hline Crop (Barley vs Wheat) & 1 & 0.809884 & 0.809884 & 94.59 & $<.001$ \\
\hline $\begin{array}{l}\text { Disease Status: Disease Class (Fusarium vs } \\
\text { Yellow rust) }\end{array}$ & 1 & 10.26827 & 10.26827 & 1199.23 & $<.001$ \\
\hline Disease Status:Water & 1 & 0.273841 & 0.273841 & 31.98 & $<.001$ \\
\hline Disease Status:Crop & 1 & 0.233846 & 0.233846 & 27.31 & $<.001$ \\
\hline Water:Crop & 1 & 0.053444 & 0.053444 & 6.24 & 0.02 \\
\hline Disease Status:Disease Class:Water & 1 & 0.054515 & 0.054515 & 6.37 & 0.019 \\
\hline Disease Status:Disease Class:Crop & 1 & 0.323653 & 0.323653 & 37.8 & $<.001$ \\
\hline Disease Status:Water:Crop & 1 & 0.001909 & 0.001909 & 0.22 & 0.641 \\
\hline Disease Status:Disease Class:Water:Crop & 1 & 0.051774 & 0.051774 & 6.05 & 0.022 \\
\hline Residual & 24 & 0.205497 & 0.008562 & 1.05 & \\
\hline \multicolumn{6}{|l|}{ sqrt(SQdiff) } \\
\hline Disease Status (Healthy vs Infected) & 1 & 0.118056 & 0.118056 & 12.66 & 0.002 \\
\hline Water (Watered vs Water stressed) & 1 & 0.000618 & 0.000618 & 0.07 & 0.799 \\
\hline Crop (Barley vs Wheat) & 1 & 0.07096 & 0.07096 & 7.61 & 0.011 \\
\hline $\begin{array}{l}\text { Disease Status:Disease Class (Fusarium vs Yellow } \\
\text { rust) }\end{array}$ & 1 & 0.310476 & 0.310476 & 33.29 & $<.001$ \\
\hline Disease Status:Water & 1 & 0.000456 & 0.000456 & 0.05 & 0.827 \\
\hline Disease Status:Crop & 1 & 0.013211 & 0.013211 & 1.42 & 0.246 \\
\hline Water:Crop & 1 & 0.001336 & 0.001336 & 0.14 & 0.708 \\
\hline Disease Status:Disease Class:Water & 1 & 0.015536 & 0.015536 & 1.67 & 0.209 \\
\hline Disease Status:Disease Class:Crop & 1 & 0.092105 & 0.092105 & 9.88 & 0.004 \\
\hline Disease Status:Water:Crop & 1 & 0.012195 & 0.012195 & 1.31 & 0.264 \\
\hline Disease Status:Disease Class:Water:Crop & 1 & 0.012502 & 0.012502 & 1.34 & 0.258 \\
\hline Residual & 24 & 0.22381 & 0.009325 & 5.08 & \\
\hline
\end{tabular}


Table 7: Summary of model prediction performance for yellow rust and fusarium head blight $\%$ coverage in wheat and barley in cross-validation and prediction. Results are shown for the determination coefficients $\left(R^{2}\right)$, root mean square error of the prediction (RMSEP) and cross validation (RMSECV), and the ratio of prediction deviation (RPD), which is the standard deviation divided by RMSEP

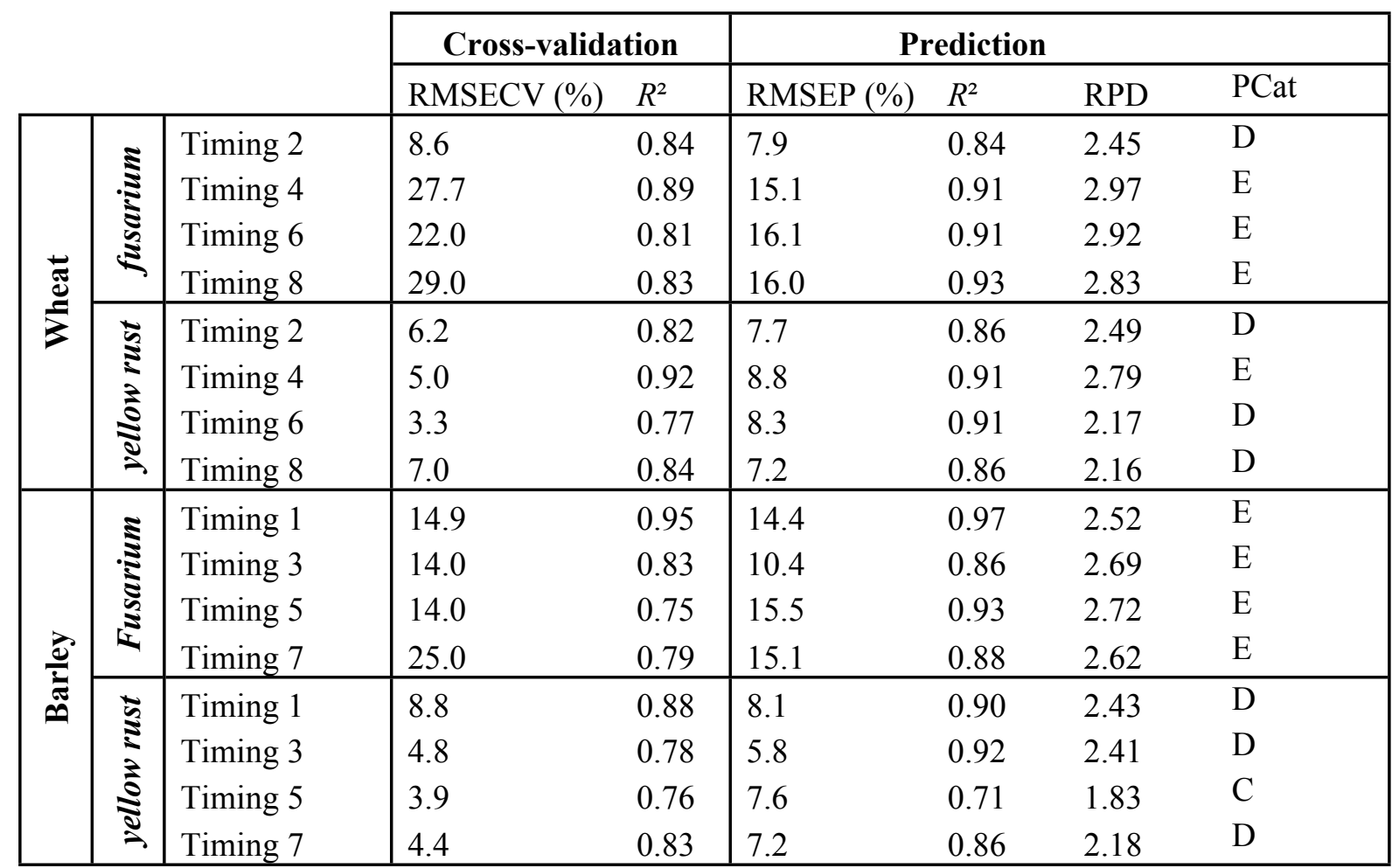

$\mathrm{PC}$ at timings in prediction category, to those detailed in Table 4. 